\title{
Structural basis of phosphatidylinositol 3-kinase C2 $\alpha$ function
}

\author{
Wen-Ting Lo $\oplus^{1 凶}$, Yingyi Zhang ${ }^{2,3,9,10}$, Oscar Vadas $\oplus^{4,10}$, Yvette Roske ${ }^{5}$, Federico Gulluni ${ }^{6}$, \\ Maria Chiara De Santis ${ }^{6}{ }^{6}$, Andreja Vujicic Zagar ${ }^{7}$, Heike Stephanowitz' ${ }^{1}$ Emilio Hirsch ${ }^{6}$, Fan Liu ${ }^{1} 1$, \\ Oliver Daumke $\oplus^{5}$, Misha Kudryashev $\circledast^{2,3}$ and Volker Haucke $\oplus^{1,8} \llbracket$
}

Phosphatidylinositol 3-kinase type $2 \alpha$ (PI3KC2 $\alpha$ ) is an essential member of the structurally unresolved class II PI3K family with crucial functions in lipid signaling, endocytosis, angiogenesis, viral replication, platelet formation and a role in mitosis. The molecular basis of these activities of PI3KC2 $\alpha$ is poorly understood. Here, we report high-resolution crystal structures as well as a 4.4-Å cryogenic-electron microscopic (cryo-EM) structure of PI3KC2 $\alpha$ in active and inactive conformations. We unravel a coincident mechanism of lipid-induced activation of PI3KC2 $\alpha$ at membranes that involves large-scale repositioning of its Ras-binding and lipid-binding distal Phox-homology and C-C2 domains, and can serve as a model for the entire class II PI3K family. Moreover, we describe a PI3KC2 $\alpha$-specific helical bundle domain that underlies its scaffolding function at the mitotic spindle. Our results advance our understanding of PI3K biology and pave the way for the development of specific inhibitors of class II PI3K function with wide applications in biomedicine.

$\mathrm{P}$ hosphoinositide 3-kinases (PI3Ks) are a family of lipidmodifying enzymes that phosphorylate the $3^{\prime}-\mathrm{OH}$ group of inositol phospholipids and play key roles in physiology ranging from cell growth and metabolism to organismal development. Dysfunction of PI3K signaling is implicated in human diseases including cancer, immunodeficiency, diabetes and neurological disorders $^{1-3}$. Mammalian PI3Ks are grouped into three classes based on their structural organization. Class I PI3Ks are receptor-activated heterodimeric enzymes with pivotal roles in cell signaling (for example, cell growth) via synthesis of phosphatidylinositol (PI) 3,4,5-trisphosphate $\left(\mathrm{PI}(3,4,5) \mathrm{P}_{3}\right)$ at the plasma membrane ${ }^{2,4-6}$. Isoform-specific pharmacological inhibitors of class I PI3K activity have undergone clinical development as anticancer therapeutics and for the treatment of human disorders caused by PI3K pathway hyperactivation.

Complexes of Vps34, the sole class III PI3K member, produce PI 3-phosphate (PI(3)P) in the endolysosomal system and during autophagy to regulate vesicle-mediated sorting en route to lysosomes ${ }^{1}$. Recent structural studies ${ }^{7,8}$ have enabled the development of selective Vps34 inhibitors that have been instrumental for the analysis and manipulation of class III PI3K function in autophagy and in the regulation of nutrient signaling.

The class II PI3K isoforms PI3KC2 $\alpha$, PI3KC2 $\beta$ and PI3KC2 $\gamma$ are unique in directly synthesizing PI 3,4-bisphosphate $\left(\mathrm{PI}(3,4) \mathrm{P}_{2}\right)$ from PI 4-phosphate $(\mathrm{PI}(4) \mathrm{P})$ at the plasma membrane and within the endolysosomal system ${ }^{9,10}$, in addition to synthesis of PI 3-phosphate (PI(3)P $)^{11-14}$. The mechanistic basis for the ability of class II PI3Ks to recognize PI(4)P as a substrate to directly produce local pools of $\mathrm{PI}(3,4) \mathrm{P}_{2}$ at defined endocytic membrane nanodomains is unknown. PI3KC2 $\alpha$ is essential in mice ${ }^{13}$. Loss of its catalytic activity is associated with cellular defects in endocytosis ${ }^{15,16}$, angiogenesis and endothelial cell function ${ }^{17-19}$, regulation of blood pressure $^{20}$, viral replication ${ }^{21,22}$, platelet formation ${ }^{23,24}$ and primary cilia signaling ${ }^{13}$. Abrogation of PI3KC2 $\alpha$ activity in animal models and in humans leads to kidney cyst formation, skeletal abnormalities, neurological symptoms and cataract formation ${ }^{25}$. In addition to these catalytic roles, PI3KC2 $\alpha$ is also required for genome stability by acting as a scaffold at the mitotic spindle during cell division ${ }^{26}$.

In contrast to class I, ${ }^{4,6}$ and class III PI3Ks (refs. ${ }^{4,7,8,27}$ ) that are understood in structural detail, little is known about the structural and functional architecture, and mechanism of activation of class II PI3Ks including PI3KC2 $\alpha$. Unlike their class I and class III relatives that are targeted to their site of action via associated subunits, class II enzymes such as PI3KC2 $\alpha$ lack stable association with other subunits $^{4,14}$ and, thus, must be activated via a distinct regulatory mechanism that so far has remained elusive. The lack of structural information on PI3KC2 $\alpha$ and related class II PI3Ks has also greatly hampered the development of isoform-selective pharmacological inhibitors for clinical applications. Moreover, the molecular basis of the scaffolding function of PI $3 \mathrm{KC} 2 \alpha$ at the mitotic spindle via its association with the microtubule-binding protein TACC 3 at kinetochore fibers to prevent aneuploidy ${ }^{26}$ is unknown.

To address these important unresolved issues, we have determined high-resolution crystal structures as well as a $4.4-\AA$ cryogenic- electron microscopic (cryo-EM) structure of PI3KC2 $\alpha$ in different conformational states and in the presence of nonselective small molecule inhibitors. Our results identify a hitherto unknown coincident mechanism of lipid-induced activation of PI3KC2 $\alpha$ that is distinct from that of all other PI3Ks and can serve as a model for the entire class II PI3K family. Moreover, our structural and

\footnotetext{
'Leibniz-Forschungsinstitut für Molekulare Pharmakologie (FMP), Berlin, Germany. ${ }^{2}$ Max Planck Institute for Biophysics, Frankfurt am Main, Germany. ${ }^{3}$ Buchmann Institute for Molecular Life Sciences, Goethe University, Frankfurt am Main, Germany. ${ }^{4}$ University of Geneva, Faculty of Medicine, Geneva, Switzerland. ${ }^{5}$ Max Delbrück Centre for Molecular Medicine (MDC), Crystallography, Berlin, Germany. ${ }^{6}$ Department of Molecular Biotechnology and Health Sciences, University of Torino, Torino, Italy. ${ }^{7}$ University of Geneva, Section of Pharmacy, Geneva, Switzerland. ${ }^{8}$ Department of Biology, Chemistry, Pharmacy, Freie Universität Berlin, Berlin, Germany. ${ }^{9}$ Present address: Biological Cryo-EM Center, Hong Kong University of Science and Technology, Clear Water Bay, Kowloon, Hong Kong, China. ${ }^{10}$ These authors contributed equally: Yingyi Zhang, Oscar Vadas. ${ }^{凶}$-mail: lo@fmp-berlin.de; haucke@fmp-berlin.de
} 
functional biochemical data will greatly facilitate the future development of isoform-selective PI3KC2 $\alpha$ inhibitors with biomedical applications that range from the treatment of thrombosis ${ }^{28}$ to viral infections ${ }^{21,22}$, diabetes ${ }^{29}$ and cancer ${ }^{1,14}$.

\section{Results}

Overall architecture of PI3KC2 $\alpha$. Like other members of the PI3K family ${ }^{1,4}, \mathrm{PI} 3 \mathrm{KC} 2 \alpha$ contains a PI3K core that consists of a Ras-binding domain (RBD) and an N-terminal C2 domain, as well as helical and kinase domains (KDs). In addition, PI3KC2 $\alpha$ harbors the C-terminal Phox-homology (PX) and C2 domains that are unique to class II enzymes (Fig. 1a). To determine the X-ray crystal structure of PI3KC2 $\alpha$, we embarked on an iterative process of construct screening and optimization of various forms of PI3KC2 $\alpha$ from different species assisted by hydrogen/deuterium exchange-mass spectrometry (HDX-MS) to identify disordered regions. A purified mouse PI3KC2 $\alpha$ construct containing a re-engineered internal loop sequence and lacking the intrinsically disordered $\mathrm{N}$-terminal region and the C-terminal C2 domain (C-C2) (Extended Data Figs. 1a,b and 2) was used to obtain the $3.3 \AA$ crystal structure of PI3KC2 $\alpha^{\Delta \mathrm{N}+\Delta \mathrm{C}-\mathrm{C} 2}$ (Table 1).

The overall structure of PI3KC2 $\alpha^{\Delta \mathrm{N}+\Delta \mathrm{C}-\mathrm{C} 2}$ comprises a large compact multidomain kinase core that is mounted onto a helical stalk (Fig. 1b). The latter is unique to class II PI3Ks and has not been observed or predicted before in any other PI3K, that is $\mathrm{PI} 3 \mathrm{~K} \alpha-\delta$ or Vps34 (Fig. 1c). The compact core region is flanked by the distantly located PX domain that is stabilized by contacts with a neighboring molecule in the crystal. Earlier biochemical data demonstrated an autoinhibitory role of the PX-C2 domains for PI3KC2 $\alpha$ catalytic activity $^{30}$. The release of the PX-C2 domain from the catalytic core may thus indicate that the obtained PI $3 \mathrm{KC} 2 \alpha^{\Delta \mathrm{N}+\Delta \mathrm{C}-\mathrm{C} 2}$ crystal structure represents an active conformation of the enzyme, a hypothesis tested and corroborated below. The core region and the helical stalk comprise an N-terminal helix and five additional domains: the RBD, the N-terminal C2 domain (N-C2), the solenoid helical domain that tightly packs against the catalytic $\mathrm{KD}$ comprising a smaller $\mathrm{N}$-terminal and a larger $\mathrm{C}$-terminal lobe, and a helical bundle domain (HBD) (Fig. 1b). The helical domain in this assembly acts as the central hub that connects the conserved archetypical two-lobed KD of PI3KC2 $\alpha$ (refs. ${ }^{4,6,31}$ ) with the N-C2 domain. The globular N-terminal RBD displays a conserved $\alpha / \beta$ sandwich fold that resembles the RBD of class I PI3Ks (refs. ${ }^{4,6}$ ) (Fig. 1c) and forms close contacts with the N-terminal lobe of the KD. In spite of this structural conservation, the primary sequence between the RBDs of PI3KC2 $\alpha$ and class I PI3Ks is poorly conserved $(<25 \%$, Extended Data Fig. 2). This is consistent with the fact that class II PI3Ks including PI3KC2 $\alpha$ functionally associate with endosomal Rab family proteins ${ }^{12,13}$ rather than with Ras, a major activator of class I PI3K $\alpha$ (refs. ${ }^{5,6,32}$ ).

A conserved feature of all mammalian class II PI3Ks is a sequence insertion of about 100 amino acids between the RBD and the N-C2 domain (Extended Data Fig. 2) that is absent in class I and class III enzymes (Fig. 1c). This sequence folds into a unique stalk-forming HBD comprising four helices (Fig. 1b). The HBD points away from the kinase core and is dispensable for catalytic activity (Extended Data Fig. 1c), suggesting that it exhibits a structural role as a protein interaction scaffold, as corroborated below (Fig. 6). The HBD contacts the N-terminal C2 domain via its $\mathrm{C}$-terminal helices and connects to this domain via a short flexible linker. The $\mathrm{N}-\mathrm{C} 2$ domain preserves an antiparallel $\beta$-sandwich topology. Based on the abundance of conserved basic residues within the disordered loop that connects $\beta$-strands 3 and 4, the N-C2 domain might associate with negatively charged membrane phospholipids. This role of the $\mathrm{N}-\mathrm{C} 2$ domain is distinct from its function in Vps34, where it serves as a protein interaction hub $^{7}$ (Fig. 1c).
Structural basis for PI3KC2 $\alpha$ catalytic activity. To gain insights into the mechanism of catalysis and to enable the development of small molecule inhibitors of PI3KC2 $\alpha$, we determined crystal structures of the kinase core domain. Based on the PI3KC $2 \alpha^{\Delta \mathrm{N}+\Delta \mathrm{C}-\mathrm{C} 2}$ construct, we designed a recombinant PI3KC $2 \alpha^{\text {core }}$ variant that lacks the N-terminal low complexity region and the distal PX and C-C2 domains. The HBD was substituted by a short seven residue-long linker (Fig. 1a and Extended Data Fig. 2). The 2.5- $\AA$ crystal structure of PI3KC $2 \alpha^{\text {core }}$ determined in the absence or presence of $\mathrm{Mg}^{2+}$ and ATP confirmed the architecture of the PI3KC2 $\alpha$ catalytic core (Fig. 2a, Extended Data Fig. 3a and Table 1) and revealed important insights into the catalytic mechanism (Fig. 2b,c).

The PI3KC2 $\alpha$ KD displays a typical PI3K KD fold comprising a smaller $\mathrm{N}$ - and a larger C-lobe linked by a hinge (Extended Data Fig. 3b). The ATP-binding P-loop is located between strands $\mathrm{k} \beta 1$ and $\mathrm{k} \beta 2$, and contacts the $\alpha$-phosphate via a conserved serine (S1114). The catalytic loop located between $\mathrm{k} \alpha 6$ and $\mathrm{k} \beta 7$ contains a conserved ${ }^{1250} \mathrm{DRH}^{1252}$ motif, in which R1251 stabilizes the $\mathrm{N}$-terminal part of the activation loop containing the ${ }^{1268} \mathrm{DFG}^{1270}$ motif. H1252 within the DRH motif could act as catalytic base in lipid kinases to deprotonate the $3^{\prime}-\mathrm{OH}$ of the inositol substrate, a reaction aided by D1250 and D1268 (ref. ${ }^{31}$ ). An atypical feature of the crystallized PI3KC2 $\alpha$ catalytic core is the presence of only a single $\mathrm{Mg}^{2+}$ ion that is coordinated by the $\beta$ - and $\gamma$-phosphates of ATP and D1146 in k $\alpha 3$ and D1268 in the DFG motif. In this $\mathrm{Mg}^{2+}$-bound state, $\mathrm{N} 1255$, that is a residue bound to a second $\mathrm{Mg}^{2+}$ ion in PI3K $\gamma^{5}$ and other PI3Ks ${ }^{4}$, interacts with D1250 and H1252 in the HRD motif, apparently to inhibit the catalytic function (Fig. $2 \mathrm{c}$ and Extended Data Fig. 3b). Hence, this conformation of PI3KC2 $\alpha$ likely reflects an early precatalytic state. Binding of the second $\mathrm{Mg}^{2+}$ conceivably induces a conformational change of N1255 to release the HRD motif from intramolecular inhibition, thereby enabling catalysis.

Class II PI3Ks including PI3KC2 $\alpha$ among other features are distinguished from class I and class III enzymes by their substrate selectivity, most notably their unique ability to use PI(4)P as a substrate to directly produce $\mathrm{PI}(3,4) \mathrm{P}_{2}$ at endocytic membranes ${ }^{1,14}$, for example to facilitate endocytosis ${ }^{15,33}$. We confirmed the preference of purified recombinant $\mathrm{PI} 3 \mathrm{KC} 2 \alpha$ to synthesize $\mathrm{PI}(3,4) \mathrm{P}_{2}$ from PI(4)P over PI(3)P synthesis from PI (Fig. 2e), in agreement with our earlier cell-based data ${ }^{15,16}$. Cellular production of $\mathrm{PI}(3,4) \mathrm{P}_{2}$ versus $\mathrm{PI}(3) \mathrm{P}$ in addition to substrate availability is likely modulated by the specific membrane environment, explaining the distinct functions of PI3KC2 $\alpha$ at the plasma membrane and at endosomes ${ }^{1,14}$. Lipid substrate binding in PI 3-kinases is encoded within the activation loop. The primary sequence of the activation loop is highly conserved also among class II PI3Ks, suggesting that they bind their substrates via similar mechanisms (Extended Data Fig. 2). While the N-terminal part of the activation loop harboring the catalytic DFG motif is clearly resolved, the C-terminal part of the PI3KC2 $\alpha$ activation loop containing the phospholipid headgroup-binding basic residues remains disordered. We therefore used the class I $\mathrm{PI} 3 \mathrm{~K} \alpha$ complexed to $\mathrm{PI}(4,5) \mathrm{P}_{2}$ (ref. ${ }^{34}$ ) as a reference to model $\mathrm{PI}(4)$ $\mathrm{P}$ headgroup binding to $\mathrm{PI} 3 \mathrm{KC} 2 \alpha$. In this model, the $3^{\prime}-\mathrm{OH}$ group of inositol is oriented toward the $\gamma$-phosphate of ATP, whereas the 4-phosphate binds to a flexible positively charged surface created by K1283 and R1284 on the C-terminal activation loop (Fig. 2d). We tested this model experimentally by creating charge neutralization mutants of PI3KC2 $\alpha$. Consistently, we found that alanine substitution of K1283 and R1284 abrogated catalytic activity toward PI(4) P (Fig. 2f) and greatly reduced phosphorylation of PI (Extended Data Fig. 3c), whereas alanine substitution of K1283 only had very minor effects.

These data provide a firm structural basis for the unique ability of PI3KC2 $\alpha$ and related class II PI3Ks to directly synthesize PI $(3,4)$ $\mathrm{P}_{2}$ from $\mathrm{PI}(4) \mathrm{P}$ to control its biological function and activity. 
a

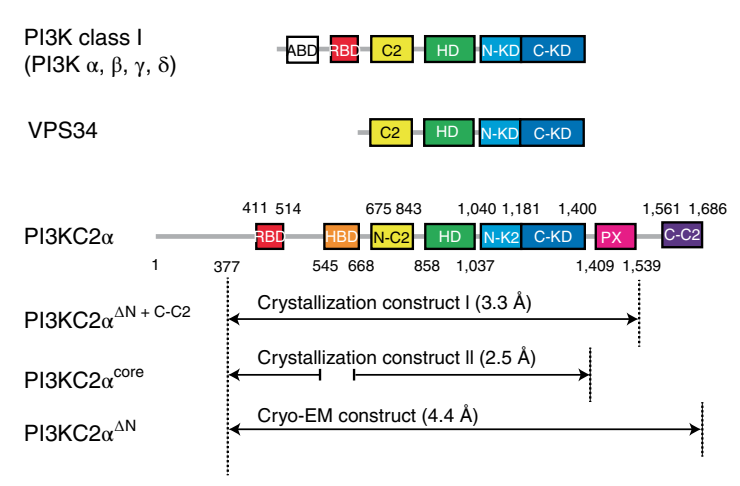

b

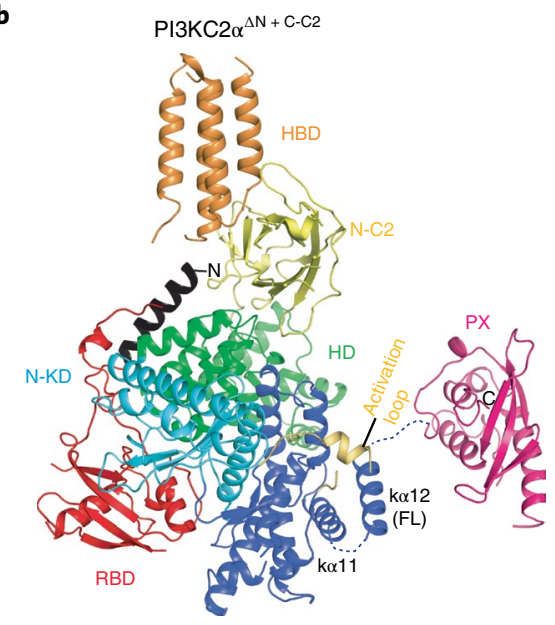

c

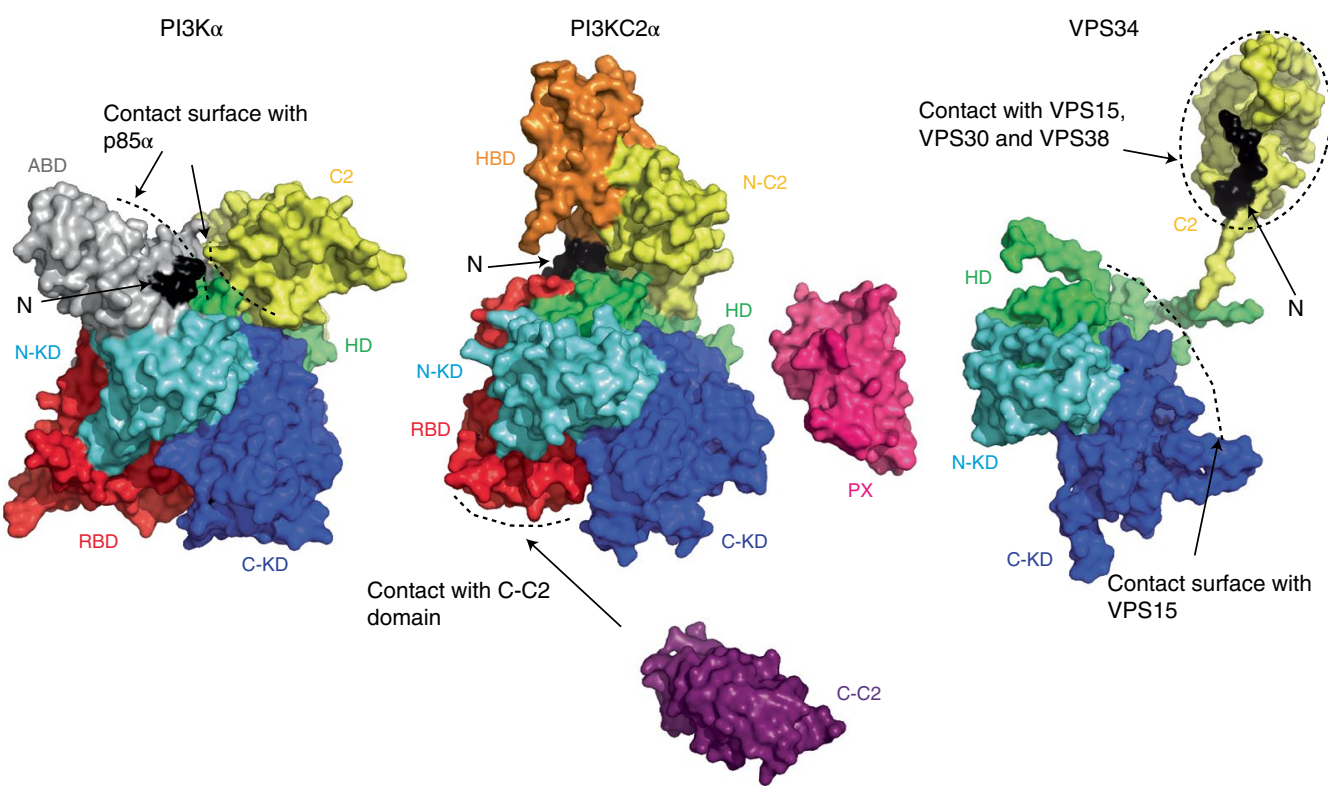

Fig. 1 | Overall structure of PI3KC2 $\boldsymbol{\alpha}$. a, Domain organization of PI3Ks. ABD, adapter binding domain, C2, C2 domain, HD, helical domain, N-KD, C-KD, $\mathrm{N}$ - and C-terminal lobes of the kinase core domain. Amino acids 1-377 of PI3KC2 $\alpha$ are predicted to be disordered. HBD specific to class II PI3Ks identified in this study. Recombinant PI3KC2 $\alpha$ constructs used for X-ray protein crystallography and single-particle cryo-EM are indicated. $\mathbf{b}$, Overall structure of PI3KC2 $\alpha^{\Delta \mathrm{N}+\Delta \mathrm{C}-\mathrm{C2}}$. The compact core comprises an N-terminal helix (black), the RBD (red), N-C2 (yellow), HD (green), N-KD (cyan) and C-KD (blue). The HBD (orange) points away from the compact core region and forms the stalk of the inverted lollipop. In this open conformation, a short helix is observed in the activation loop (yellow) and the k $\alpha 12$ helix within the KD is well folded. The N terminus of the PX domain is located $15.6 \AA$ away from the $C$ terminus

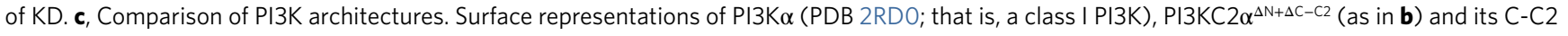
domain (PDB 6BTY) (that is, a class II PI3K), and Vps34 (PDB 5DFZ; that is, class III PI3K). The HD and N-KD of PI3K $\alpha$ contact the N-terminal domain of $\mathrm{PI} 3 \mathrm{~K} \alpha$; the $\mathrm{p} 85 \alpha$ regulatory subunit associates with the ABD. PI3KC2 $\alpha$ lacks regulatory subunits but contains a unique HBD as well as lipid-binding PX and C-C2 domains that regulate membrane binding and activity. In Vps34 the N-terminal C2 domain acts as a protein interaction hub for its associated subunits Vps15, Vps30 and Vps38.

Small molecule inhibition of PI3KC2 $\alpha$ catalytic function. Given the multiple important roles of PI3KC2 $\alpha$ and related class II PI3Ks in cell physiology and in disease ${ }^{1,14}$, we next sought to probe the structural basis for inhibition of its catalytic activity by small molecules. Although no specific inhibitors of PI3KC2 $\alpha$ have been identified so far, the enzyme is known to be targeted by pan-PI 3-kinase inhibitors (that is, PIK-90, Extended Data Fig. 4b) ${ }^{35}$ and by Torin2, an ATP-competitive inhibitor of the PI3K-related kinase superfamily member mTOR $^{36}$ (Extended Data Fig. 4a). To explore the determinants of inhibitor potency and specificity, we determined the structures of PI3KC $2 \alpha^{\text {core }}$ bound to Torin- 2 or PIK- 90 and compared these to the structure of the apo-form of the enzyme (Fig. 3a).
Torin-2 binds to PI3KC2 $\alpha$ in a mode similar to mTOR (Fig. $3 \mathrm{~b}$ and Extended Data Fig. 4c). The tricyclic benzonapththyridine ring of Torin-2 occupies the hydrophobic adenine pocket and a sulfur-pi interaction contributed by the conserved M1125 located in hydrophobic pocket II. Binding is further enabled by local small scale conformational changes of K1138 and D1268 to accommodate the amino-pyrimidine group of Torin- 2 and by hydrophobic contacts of the benzotrifluoride group with M1136 and F1112 (Fig. 3b,c). The reduced half-maximum inhibitory concentration $\left(\mathrm{IC}_{50}\right)$ of Torin-2 for $\mathrm{mTOR}\left(\mathrm{IC}_{50}{ }^{\mathrm{mTOR}}=2.5 \mathrm{nM}\right.$ versus $\left.\mathrm{IC}_{50}{ }^{\mathrm{PI} 3 \mathrm{KC} 2 \alpha}=64 \mathrm{nM}\right)$ compared to $\mathrm{PI} 3 \mathrm{KC} 2 \alpha$ is largely due to the pi-pi interaction of the tricyclic benzonapththyridine ring with W2239 inside the mTOR hinge 
Table 1 | Data collection and refinement statistics

\begin{tabular}{|c|c|c|c|c|c|}
\hline & $\mathrm{PI} 3 \mathrm{KC} 2 \alpha^{\Delta \mathrm{N}+\mathrm{C}-\mathrm{C} 2}$ & PI3KC2 $\alpha^{\text {core }}($ apo) & PI3KC $2 \alpha^{\text {core }}\left(\right.$ ATP- $\left.\mathrm{Mg}^{2+}\right)$ & PI3KC2 $\alpha^{\text {core }}$ (Torin-2) & PI3KC2 $\alpha^{\text {core }}($ PIK-90) \\
\hline \multicolumn{6}{|l|}{ Data collection ${ }^{\mathrm{a}}$} \\
\hline Space group & $P 2_{1} 2_{1} 2_{1}$ & $P 2_{1} 2_{1} 2_{1}$ & $P 2_{1} 2_{1} 2_{1}$ & $P 2_{1} 2_{1} 2_{1}$ & $P 2_{1} 2_{1} 2_{1}$ \\
\hline$a, b, c(\AA)$ & $82.6,115.9,144.4$ & $135.2,151.6,56.0$ & $56.1,133.4,152.7$ & $55.3,134.2,151.0$ & $55.9,135.4,151.6$ \\
\hline$\alpha, \beta, \gamma\left(^{\circ}\right)$ & $90.0,90.0,90.0$ & $90.0,90.0,90.0$ & $90.0,90.0,90.0$ & $90.0,90.0,90.0$ & $90.0,90.0,90.0$ \\
\hline Resolution $(\AA)$ & $49.20-3.25(3.45-3.25)^{b}$ & $43.20-2.42(2.51-2.42)$ & $47.56-2.75(2.85-2.75)$ & $47.49-2.59(2.68-2.59)$ & $42.26-2.65(2.75-2.65)$ \\
\hline$|/ \sigma|$ & $9.73(0.84)$ & $10.56(0.60)$ & $9.58(0.77)$ & $10.91(0.67)$ & $11.17(0.72)$ \\
\hline Completeness (\%) & $99.1(97.1)$ & $99.6(99.0)$ & $99.5(99.4)$ & $97.6(93.4)$ & $97.6(96.2)$ \\
\hline Redundancy & $4.9(4.9)$ & $4.5(4.4)$ & $4.2(4.1)$ & $4.1(3.8)$ & $4.0(3.8)$ \\
\hline \multicolumn{6}{|l|}{ Refinement } \\
\hline Resolution $(\AA)$ & 3.25 & 2.42 & 2.75 & 2.59 & 2.65 \\
\hline Protein & 8,224 & 6,552 & 6,611 & 6,519 & 6,540 \\
\hline Ligand/ion & 41 & 13 & 50 & 65 & 46 \\
\hline Water & 19 & 85 & 86 & 79 & 56 \\
\hline \multicolumn{6}{|l|}{$B$ factors } \\
\hline Protein & 136.62 & 72.15 & 82.14 & 86.56 & 80.84 \\
\hline Ligand/ion & 231.63 & 106.22 & 100.98 & 118.78 & 98.83 \\
\hline Water & 139.07 & 62.28 & 59.11 & 74.12 & 61.86 \\
\hline \multicolumn{6}{|l|}{ R.m.s. deviations } \\
\hline Bond lengths ( $\AA$ ) & 0.013 & 0.009 & 0.014 & 0.004 & 0.013 \\
\hline
\end{tabular}

${ }^{a}$ One crystal for each structure was used for data collection and structure determination. ${ }^{b}$ Values in parentheses are for the highest-resolution shell.

region $^{36}$, a residue not conserved in PI3KC2 $\alpha$ (Fig. $3 \mathrm{~b}$ and Extended Data Fig. 4c). PIK-90, which also displays profound off-target activity toward $\mathrm{PI} 3 \mathrm{KC} 2 \alpha\left(\mathrm{IC}_{50}^{\mathrm{PI} 3 \mathrm{KC} 2 \alpha}=78 \mathrm{nM}\right)$, occupies a similar position to Torin-2 in the ATP site. The imidazoquinazoline ring of PIK-90 binds to the adenine pocket via a single hydrogen bond and hydrophobic region II (Fig. 3d and Extended Data Fig. 4d), while the pyridine ring in the terminal hinge of PIK-90 targets the innermost region of the affinity pocket (Fig. $3 \mathrm{~d}, \mathrm{e})$. In contrast to Torin2 , association of PI3KC2 $\alpha$ with PIK-90 involves comparably minor conformational movements of K1138 and D1268 (Fig. 3e). We conclude that Torin- 2 and PIK-90 bind to PI3KC2 $\alpha$ via the conserved ATP-binding site common to PI3K-related kinases and PI3Ks and in a manner similar to complex formation with their target enzymes (Extended Data Fig. 4c,d), providing a structural basis for their high affinity but moderate selectivity.

Based on these data and the size and chemical composition of the catalytic site in $\mathrm{PI} 3 \mathrm{KC} 2 \alpha$ we predict that PI3KC $2 \alpha$ should in principle be amenable to selective targeting by high-affinity small molecule inhibitors.

Conformational control of PI3KC2 $\alpha$ activity. Unlike class I and class III PI3Ks that are activated by membrane binding of their associated subunits $2,4,7,27,34$, class II PI3Ks such as PI3KC2 $\alpha$ are autoregulated by their lipid-binding distal PX and C2 domains ${ }^{30}$. To structurally dissect the mechanism of PI $3 \mathrm{KC} 2 \alpha$ autoregulation, we purified near full-length PI3KC2 $\alpha$ only lacking the intrinsically disordered $\mathrm{N}$-terminal region but containing the distal PX and C2 domains (PI3KC2 $\alpha^{\Delta \mathrm{N}}$ ) (Extended Data Fig. 1a,b). We then determined the three-dimensional (3D) structure of PI3KC $2 \alpha^{\Delta \mathrm{N}}$ by single-particle cryo-EM (Fig. 4 and Extended Data Fig. 5). Tilted data collection followed by two-dimensional (2D) classification resulted in a reconstruction with a nominal resolution of $4.4 \AA$ from roughly 600,000 particles (Table 2 ) with substantial resolution anisotropy and the highest resolution in the core domain (Extended Data Fig. 5). The cryo-EM structure of PI3KC $2 \alpha^{\Delta \mathrm{N}}$ unequivocally showed the catalytic core of $\mathrm{PI} 3 \mathrm{KC} 2 \alpha$, which was overlaid almost perfectly with the PI $3 \mathrm{KC} 2 \alpha$ crystal structure. Two additional EM densities were located underneath the core region: a donut-shaped density in $2 \mathrm{D}$ classes that adopted a barrel shape in the 3D map. This density could accommodate the previously solved crystal structure of the C-terminal C2 domain of PI3KC2 $\alpha$ (ref. ${ }^{37}$ ) (Fig. 4). A second, less well-defined density was located in the vicinity of the C-terminal lobe of the catalytic kinase core domain, which likely corresponds to the lipid-binding PX domain (Fig. 4).

As the PX domain exhibited significant flexibility within PI3KC2 $\alpha$, we complemented our results from single-particle cryo-EM by crosslinking-mass spectrometry (XL-MS). This analysis (Supplementary Table 2) imposed distance constraints (30 ̊, measured by the $\mathrm{C} \alpha-\mathrm{C} \alpha$ distance between two crosslinked residues) of the PX domain relative to the $\mathrm{C}-\mathrm{C} 2$ domain and the C-terminal lobe of the kinase core domain (Extended Data Fig. $6 \mathrm{a}-\mathrm{c}$ ), and supported docking of the PX domain into the cryo-EM structure ${ }^{38}$. We then used the information derived from cryo-EM, XL-MS and the crystal structure of PI3KC2 $\alpha^{\text {core }}$ to 


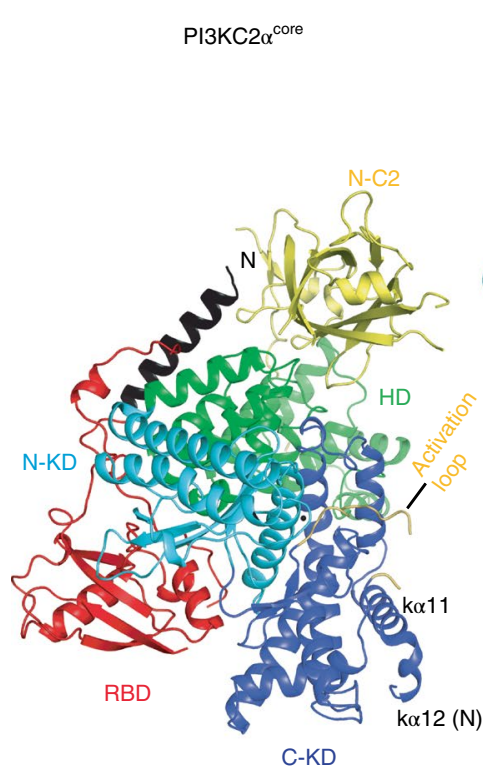

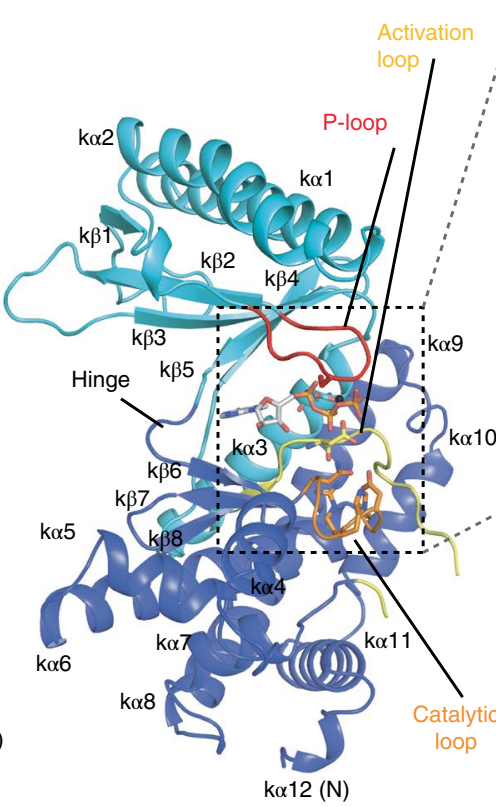

c

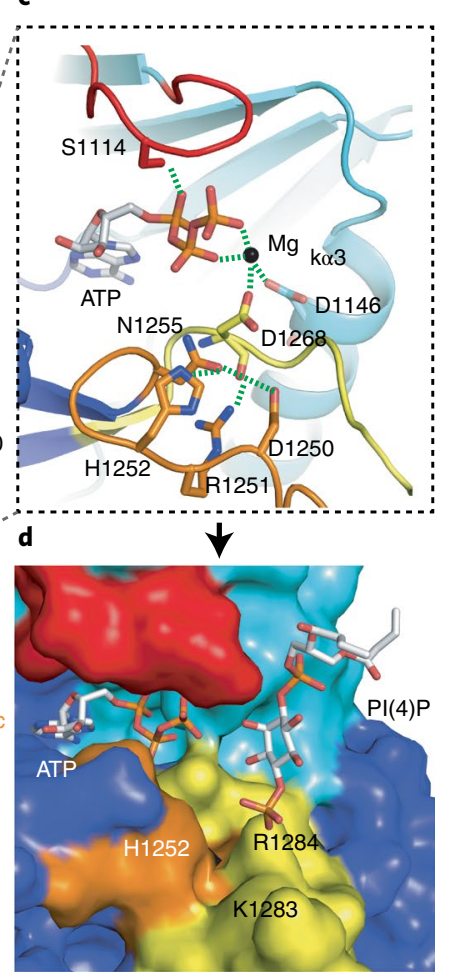

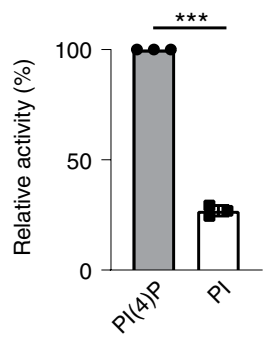

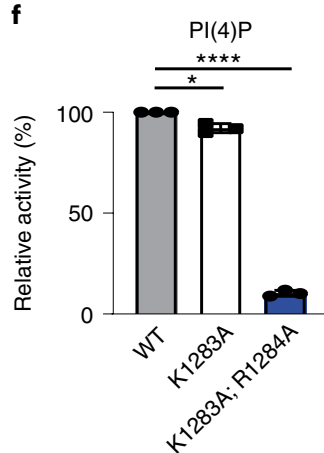

Fig. 2 | Structure and activity of the PI3KC2 $\alpha$ kinase core domain. a, Overall structure of PI3KC $2 \alpha^{\text {core }}$. Note that in this conformation, the short helix within the activation loop is disordered and only the N-terminal part of k $\alpha 12$ is folded. $\mathbf{b}$, The kinase core domain (KD) of PI3KC2 $\alpha$ in complex with ATP and $\mathrm{Mg}^{2+}$. P-loop (red), catalytic loop (orange) and activation loop (yellow) are well defined. ATP is found at the interface between the $\mathrm{N}$ and $\mathrm{C}$ lobes of the KD. c, Close-up view of the ATP-binding pocket. Green dashed lines indicate interactions. The $\mathrm{Mg}^{2+}$ ion (gray sphere) neutralizes the charges of D1146 and D1268 to enable phosphate binding of ATP (shown as sticks). R1251 in the catalytic loop interacts with the activation loop. Intramolecular interactions of D1250, H1252 and N1255 in the catalytic loop are identified. d, Model for diC4-PI(4)P binding to the KD of PI3KC2 $\alpha$. Surface representation of the $\mathrm{KD}$ with $\mathrm{K} 1283$ and R1284 in the activation loop contacting the 4-phosphate of PI(4)P. e, In vitro kinase activity of PI3KC2 $\alpha^{\Delta N}$ using either PI(4)P or PI as a substrate. Data represent mean \pm s.d. from $n=3$ experiments, one sample, two-tailed $t$-test with hypothetical mean of $100, * * * P=0.0003$. f, $P I(3,4)$ $\mathrm{P}_{2}$-synthesizing activity of PI3KC2 $\alpha^{\Delta \mathrm{N}}$ activation loop mutants. Mutation of K1283A and K1284A abrogates kinase activity. Data represent mean \pm s.d. from $n=3$ experiments, One sample, two-tailed $t$-test with hypothetical mean of $100,{ }^{*} P=0.0263$ and ${ }^{* * * *} P<0.0001$.

generate a composite 3D model of PI3KC2 $\alpha$. In this model, only the $\mathrm{N}$-terminal half of $\mathrm{k} \alpha 12(\mathrm{k} \alpha 12(\mathrm{~N}))$ is folded whereas the remainder of it is disordered. $k \alpha 12(\mathrm{~N})$ interacts with the loop between $k \alpha 7$ and $\mathrm{k} \alpha 8$ of the KD forming closed contact I. The disordered C-terminal half of k $\alpha 12$ provides the flexibility necessary to place the C-C2 domain in the vicinity of the RBD, where it forms another set of interactions referred to as closed contact II (Fig. 5a). These contacts precisely map to putative autoinhibitory interfaces defined earlier by HDX-MS analysis ${ }^{30}$. In particular, we had reported that mutation of ${ }^{1303} \mathrm{EKP}^{1305}$ to ${ }^{1303} \mathrm{KKT}^{1305}$ (that is, 'KKT mutant'), in the kinase core domain in closed contact I, results in elevated lipid kinase activity. Moreover, HDX-MS analysis had identified a putative intramolecular interaction between the RBD and the distal PX-C2 domain mod$\mathrm{ule}^{30}$. Our integrative structural analysis identifies this interaction as closed contact II, formed by K426, W458, D461 and D462 on the RBD. Based on these data, we hypothesized that the composite structure of PI3KC2 $\alpha^{\Delta \mathrm{N}}$ represents an inactive conformation that is stabilized by closed contacts I and II.

We further experimentally tested this model. Affinity chromatography experiments showed that the immobilized glutathione $S$-transferase- (GST-)tagged C2, but not the PX domain, directly associates with PI3KC $2 \alpha^{\Delta \mathrm{N}+\Delta \mathrm{PX}-\mathrm{C} 2}$ but less well with a mutant version of PI3KC2 $\alpha$, in which the RBD binding interface with the distal $\mathrm{C} 2$ domain had been perturbed by mutations in the closed contact I interface (K426A, W458A, D461A, D462A; that is, the 'RBD mutant') (Extended Data Fig. 6d). Disruption of either closed contact I in the KKT mutant $\left({ }^{1303} \mathrm{EKP}^{1305}\right.$ to $\left.{ }^{1303} \mathrm{KKT}^{1305}\right)$ or of contact II in the RBD mutant (K426A, W458A, D461A, D462A) significantly increased the $\mathrm{PI}(3,4) \mathrm{P}_{2}$-synthesizing activity of $\mathrm{PI} 3 \mathrm{KC} 2 \alpha$, a phenotype further augmented in a double KKT/RBD mutant of PI3KC2 $\alpha$, in which both inhibitory interfaces were disrupted (Fig. 5c).

As PI3KC2 $\alpha$ transitions from the inactive closed to the active open conformation, the C2 and PX domains likely are dislodged from their positions at the RBD and kinase core, respectively, while ko12 undergoes repositioning and folding into a complete helix (Fig. 5a,b). Consistently, we observed the PX domain to be displaced from the kinase core, and closed contact I involving the $\mathrm{N}$ terminus of k $\alpha 12$ to be disrupted in the crystal structure of PI3KC $2 \alpha^{\Delta N+\Delta C-C 2}$ (Fig. $1 \mathrm{~b}$ and Extended Data Fig. 3a). Instead, in this open conformation of PI3KC2 $\alpha$, the k $\alpha 12$ helix interacts via hydrogen bonding of H1391 with the backbone of a short helical segment within the intermediate section of the activation loop (Fig. 5b). In the open conformation, the C-terminal part of the activation loop known to be crucial for lipid binding remains flexible to enable catalysis.

This mechanism is distinct from the function of k $\alpha 12$ in other PI3Ks. PI3K $\gamma$ uses k 12 to capture the catalytic loop in an inactive state (Extended Data Fig. 7a), whereas in VPS34, k $\alpha 12$ interacts with the $\mathrm{N}$ terminus of VPS15 to trap the activation loop ${ }^{7}$ (Extended Data Fig. 7b). Finally, conformational opening requires the lipid-binding distal C2 domain to be displaced from its inhibitory 


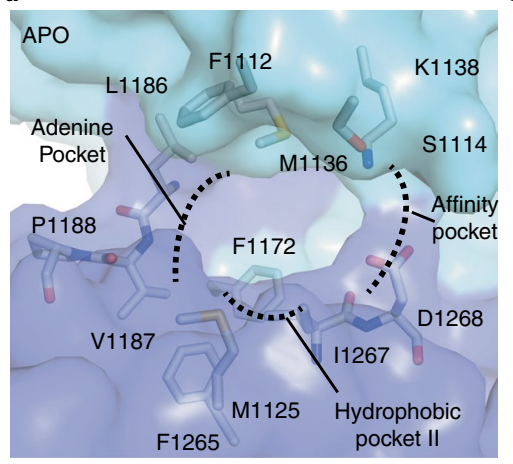

d

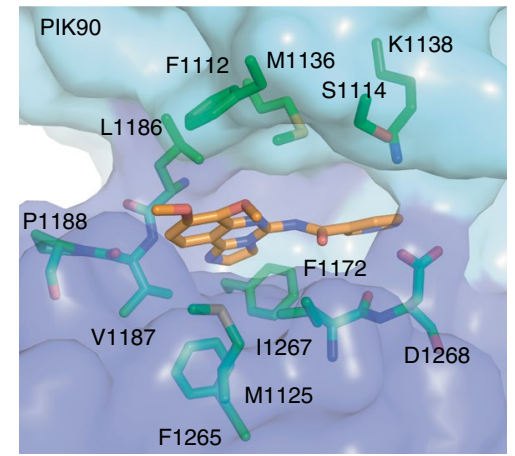

b

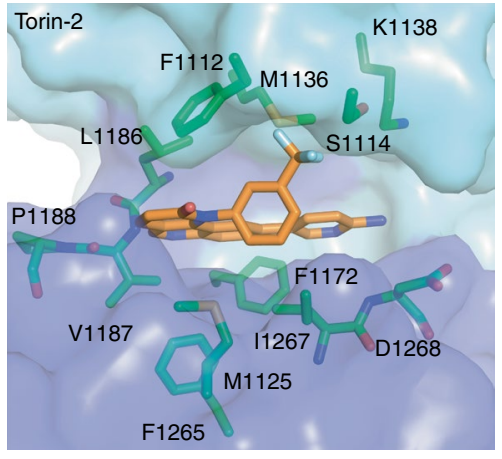

e

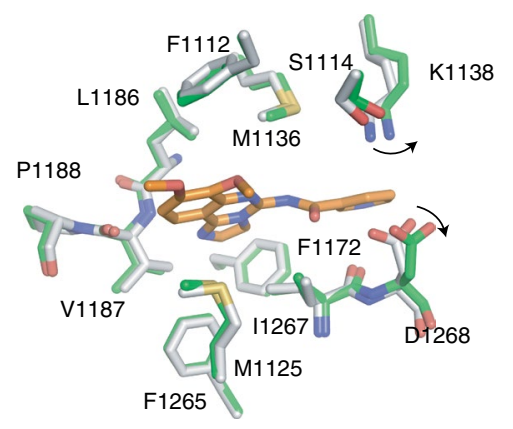

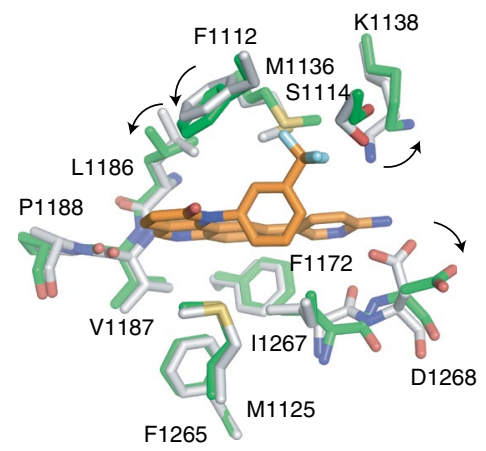

F1265
268

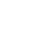

Fig. 3 | Structural basis for pharmacological inhibition of PI3KC2 $\alpha$ by Torin-2 and PIK-90. a, The ATP-binding site of the PI3KC2 $\alpha$ apo-enzyme. The adenine pocket, affinity pocket and hydrophobic pocket II are indicated with dashed line areas. $\mathbf{b}$, Torin-2 binding to the ATP-binding site. The benzonapththyridine ring occupies the adenine pocket and hydrophobic pocket II. The inner amino-pyrimidine group targets the affinity pocket. The benzotrifluoride group binds to the N-lobe of the KD (cyan). c, Comparison of the binding pocket of the apo-enzyme (gray) and the PI3KC2 $\alpha$-Torin-2 complex (green). Arrows indicate conformational changes of K1138, D1268, F1112 and L1186 elicited by Torin-2 binding. d, PIK-90 binding to the ATP-binding site. The imidazoquinazoline ring of PIK-90 occupies the adenine pocket and hydrophobic pocket II. The terminal pyridine ring binds to the inner surface of the affinity pocket. e, Comparison of the binding pocket of the apo-enzyme (gray) and the PI3KC2 $\alpha$-PIK-90 complex (green). Arrows indicate conformational changes to the inhibitor binding. PIK-90 induces comparably minor conformational changes of the binding pocket.

contact with the RBD. In the closed conformation, association with the RBD renders the lipid-binding surface of the $\mathrm{C} 2$ domain difficult to access and misaligns it with the substrate lipid-binding site in the activation loop (Extended Data Fig. 7c). Hence, we predict the distal C2 domain to be flexibly positioned away from the kinase core in the open conformation of the enzyme (Fig. 6e and below). A further prediction from this structure-based activation mechanism is that disruption of the interaction between $k \alpha 12$ and the activation loop in the open form should abrogate lipid kinase activity. In vitro kinase assays confirmed that the H1391A mutation in the center of the k $\alpha 12$-activation loop interface resulted in a complete loss of enzymatic activity (Fig. 5c). H1391 is thus required to stabilize the open catalytically active conformation of PI3KC $2 \alpha$.

Our combined data indicate a molecular model for the conformational control of PI3KC2 $\alpha$ activity by large-scale rearrangements in the position of the lipid-binding PX and C2 domains that is accompanied by refolding and repositioning of the k $\alpha 12$ helix critical for catalysis.

Conformational control of PI3KC2 $\alpha$ function in cells. We tested this structure-based model for the conformational activation of $\mathrm{PI} 3 \mathrm{KC} 2 \alpha$ at membranes by analyzing the $\mathrm{PI}(3,4) \mathrm{P}_{2}$-synthesizing activity of $\mathrm{PI} 3 \mathrm{KC} 2 \alpha$ during endocytic membrane dynamics. Depletion of PI3KC2 $\alpha$ from Cos7 cells resulted in reduced levels of $\mathrm{PI}(3,4) \mathrm{P}_{2}$ at endocytic plasma membrane coated pits and a concomitant reduction in clathrin-mediated endocytosis of transferrin. These defects were rescued by re-expression of the small interfering RNA-resistant wild-type (WT) enzyme (Fig. 5d-f) or a mutant lacking the HBD (Fig. 6a), in agreement with its presumed scaffolding role during mitosis. In contrast, $\mathrm{PI} 3 \mathrm{KC} 2 \alpha$ mutant versions defective in PI(4)P substrate binding (K1283A,R1284A) or lacking critical hydrogen bonding via H1391 to stabilize the open conformation (H1391A) failed to restore $\mathrm{PI}(3,4) \mathrm{P}_{2}$ levels and defective endocytosis. Conversely, conformational activation of PI3KC $2 \alpha$ by disrupting closed contacts I and II via the combined KKT and RBD mutations led to elevated cellular $\mathrm{PI}(3,4) \mathrm{P}_{2}$ synthesis and a gain in endocytic transferrin uptake (Fig. $5 \mathrm{~d}-\mathrm{f}$ ). These results confirm that structural changes in the position of the lipid-binding PX and C2 domains and the k $\alpha 12$ helix underlie the conformational activation of PI3KC2 $\alpha$ at membranes in vivo.

A further prediction from our combined structural and biochemical studies is that distinct structural elements mediate the catalytic roles of PI3KC2 $\alpha$ in endocytic membrane dynamics and its noncatalytic function at the mitotic spindle ${ }^{1,14}$. The unique HBD of PI3KC2 $\alpha$, which points away from the KD (Figs. $1 \mathrm{~b}$ and $4 \mathrm{c}$ ), is dispensable for catalytic activity in vitro (Extended Data Fig. 1c) and for endocytosis in vivo (Fig. 6a). We therefore hypothesized, that the HBD might facilitate targeting of the enzyme to the mitotic spindle by associating with the microtubule-binding protein TACC3 (ref. ${ }^{26}$ ). Consistently, we found that the HBD of PI3KC2 $\alpha$ with its four antiparallel $\alpha$-helices displays strong structural homology to cytoskeletal proteins, such as the focal-adhesion targeting domain of Crk-associated substrate (Cas) and to the F-actin binding domains of vinculin and $\alpha$-catenin (Fig. 6b). To probe the possible function of the HBD in targeting of PI3KC2 $\alpha$ to the mitotic spindle, we examined the subcellular localization of an N-terminally 
a

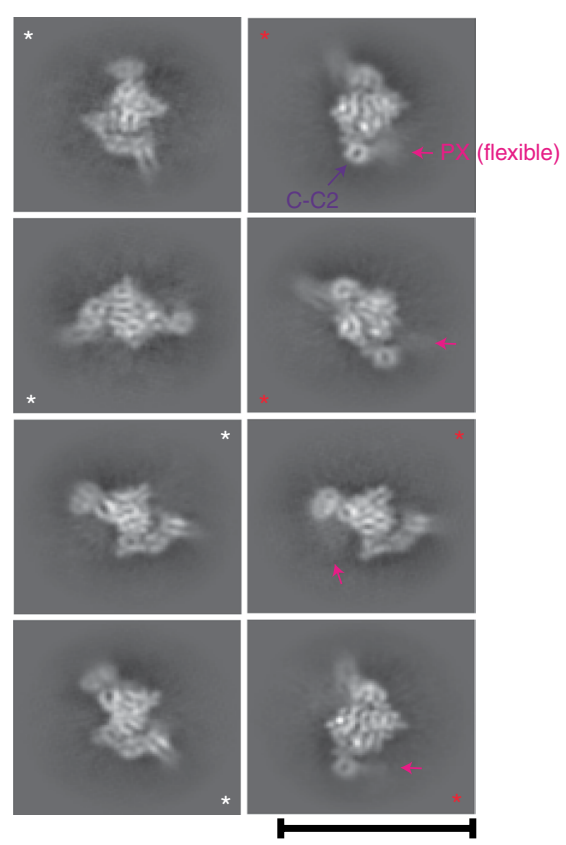

b

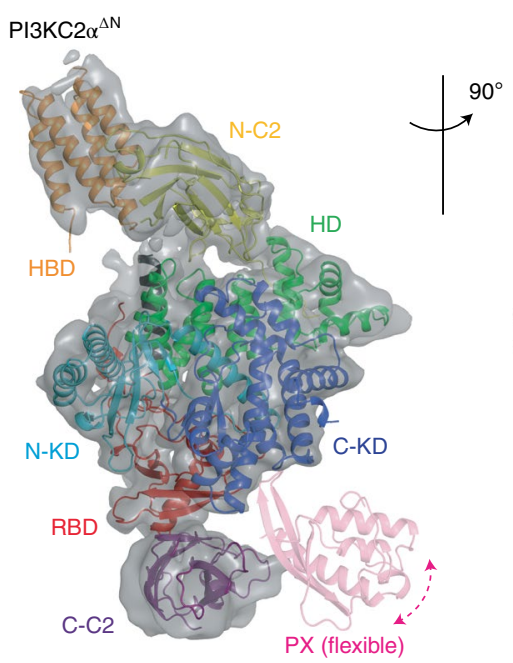

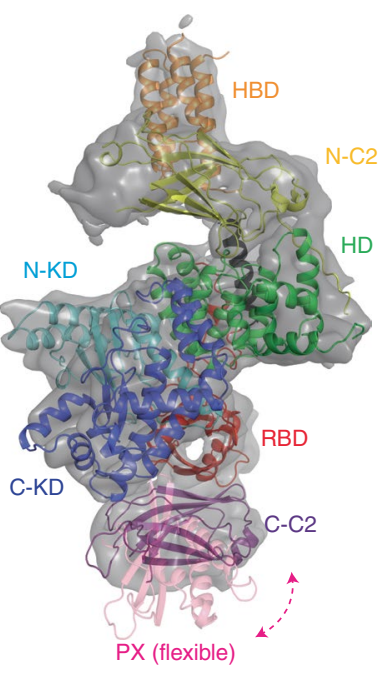

Fig. 4 | Cryo-EM structure of PI3KC2 $\alpha^{\Delta \mathbf{N}}$. a, The selected 2D classes of PI3KC2 $\alpha^{\Delta \mathrm{N}}$ from Extended Data Fig. 5b. The selected 2D classes without or with diffused density of PX domain are labeled with white or red stars. The density of PX domain is shown with a magenta arrow. The donut-shaped C-C2 domain is indicated with a purple arrow. Scale bar, $20 \mathrm{~nm}$. b,c, 3D cryo-EM map of PI3KC $2 \alpha^{\Delta \mathrm{N}}$ overlaid onto the crystal structures of PI3KC2 $\alpha^{\Delta N+\Delta C-C 2}$ and PI3KC2 $\alpha^{\text {core }}$ and the C-terminal C2 domain of human PI3KC2 $\alpha$ (PDB 6BTY). The flexible PX domain is shown as a transparent ribbon, where the EM density was subtracted after $3 \mathrm{D}$ reconstruction. The overall structure of $\mathrm{PI} 3 \mathrm{KC} 2 \alpha^{\Delta \mathrm{N}}$ reveals a closed conformation of the enzyme.The views in $\mathbf{b}$ and $\mathbf{c}$ are related by $90^{\circ}$ rotation.

truncated PI3KC2 $\alpha$ lacking the clathrin binding region, a mutant version thereof, in which the HBD was deleted (PI3KC $2 \alpha^{\Delta \mathrm{N}+\Delta \mathrm{HBD}}$ ), or the isolated HBD alone (PI3KC2 $\alpha^{\mathrm{HBD}}$ ). The HBD was sufficient for targeting to the mitotic spindle and for association with TACC3 (Fig. 6c,d), whereas deletion of the HBD abrogated the spindle localization of PI3KC2 $\alpha^{\Delta \mathrm{N}}$ (Fig. 6c) and complex formation with TACC3 (Fig. 6d). These data uncover the unique HBD as an important structural element that underlies the scaffolding function of PI3KC2 $\alpha$ at the mitotic spindle ${ }^{26}$. We note that while the presence of the HBD is conserved among the members of the class II PI3K subfamily, its relatively low level of sequence conservation (Extended Data Fig. 2) suggests that they interact with different protein binding partners to execute putative noncatalytic functions.

\section{Discussion}

Our integrated structural analysis of PI3KC2 $\alpha$ reveals different conformational states of the enzyme that suggest a molecular model for the local activation of PI3KC2 $\alpha$ at endocytic membranes (Fig. 6e). In its cytosolic form, the enzyme is present in a closed inactive conformation that is stabilized by intramolecular contacts within the kinase core domain that occlude catalysis and an inhibitory interface between the RBD and the distal C2 domain, which may be further augmented by placement of the PX domain at the interface between the distal $\mathrm{C} 2$ and the C-terminal lobe of the kinase core domain, in agreement with our earlier biochemical data ${ }^{30}$. Clathrin-mediated recruitment ${ }^{15,39}$ and activation of PI3KC2 $\alpha$ at endocytic membranes involves a large-scale conformational change within the single subunit enzyme that releases PI3KC2 $\alpha$ from autoinhibition to enable local PI 3-phosphate synthesis (Fig. 5). In this active conformation, the substrate PI(4)P is bound by basic residues (that is, K1283, R1284) within the PI3KC2 $\alpha$ activation loop (Fig. 2d-f). Of note, these residues are absent from Vps34, a PI3K that is unable to use $\mathrm{PI}(4) \mathrm{P}$ as a substrate, providing a molecular

\begin{tabular}{|lll}
\hline Table 2 | Cryo-EM data collection statistics & \\
& $\begin{array}{l}\text { PI3KC2 } \boldsymbol{\alpha} \\
\text { (untilted) } \\
\text { (EMDB-12191) }\end{array}$ & $\begin{array}{l}\text { PI3KC2 } \boldsymbol{\alpha} \\
\left(-30^{\circ} \text { tilted) }\right. \\
\text { (EMDB-12191) }\end{array}$ \\
\hline Data collection and processing & & \\
\hline Magnification & 105,000 & 105,000 \\
\hline Voltage $(\mathrm{kV})$ & 300 & 300 \\
\hline Electron exposure $\left(\mathrm{e}^{-} / \AA^{2}\right)$ & 60 & 60 \\
\hline Defocus range $(\mu \mathrm{m})$ & -1.5 to -2.8 & -1.5 to -2.8 \\
\hline Pixel size $(\AA)$ & 0.837 & 0.837 \\
\hline Symmetry imposed & $\mathrm{C} 1$ & $\mathrm{C1}$ \\
\hline Initial particle images $(\mathrm{no})$. & 2.3 million & 452,000 \\
\hline Final particle images $(\mathrm{no})$. & 601,000 (untilted + tilted) \\
\hline Map resolution $(\AA)$ & 4.4 & \\
\hline FSC threshold & 0.143 & \\
\hline Map resolution range $(\AA)$ & $5.2-4.4$ & \\
\hline
\end{tabular}

explanation for the distinct catalytic activities of PI3KC2 $\alpha$ and related class II PI3Ks (refs. ${ }^{1,2,14}$ ).

The mechanism of activation of PI3KC2 $\alpha$ is distinct from that of all other PI3Ks, in which membrane binding and catalytic activation are induced by conformational transitions in tightly associated accessory subunits (compare Fig. 1c). In class I PI3K, hydrophobic residues in the $\mathrm{C}$-terminal tail of the $\mathrm{KD}$ not present in PI3KC2 $\alpha$ as well as basic amino acids in the charged activation loop are only allowed to contact the membrane once the enzyme has been released from allosteric inhibition by its regulatory p85 subunit ${ }^{40}$. 


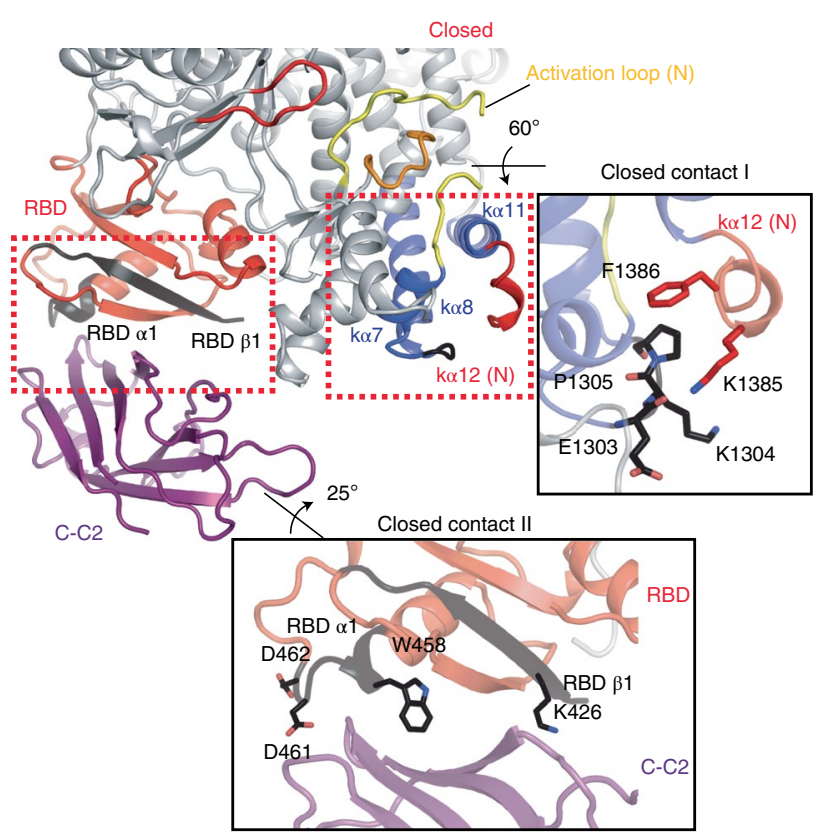

C

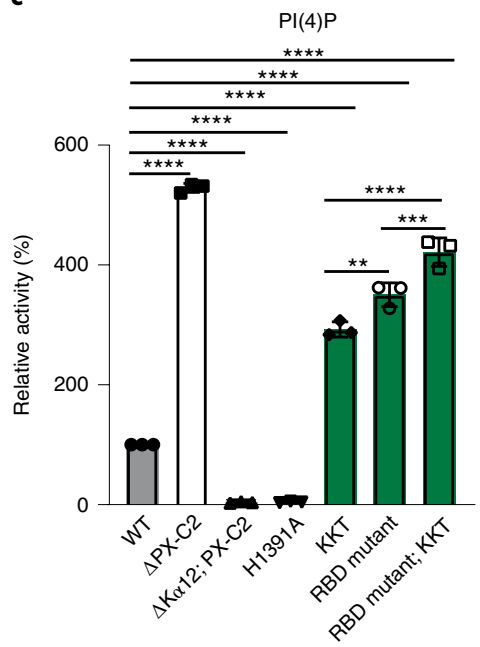

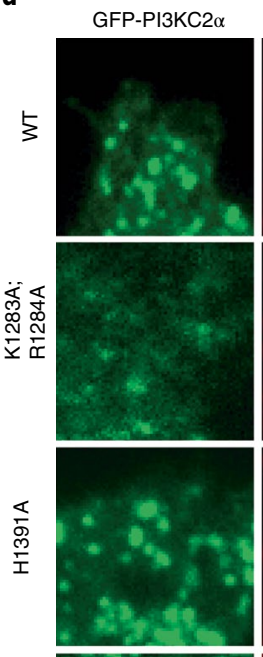

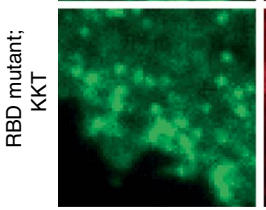

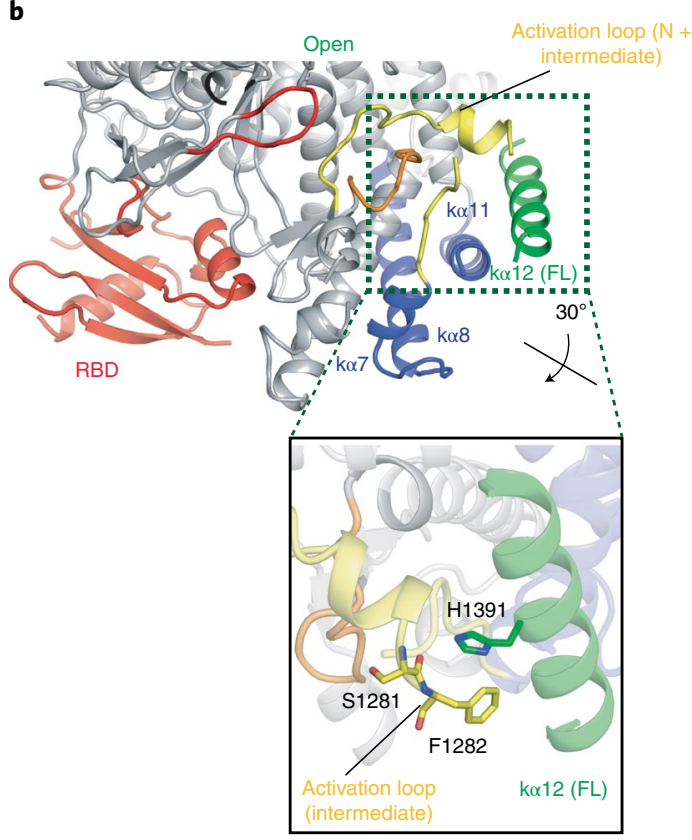
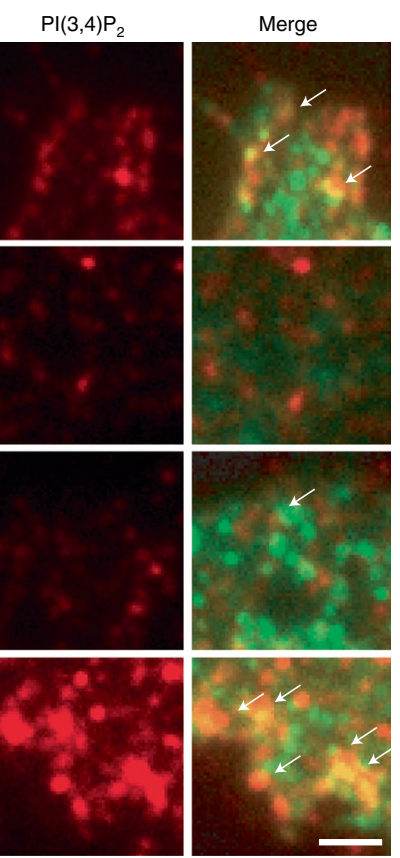

e
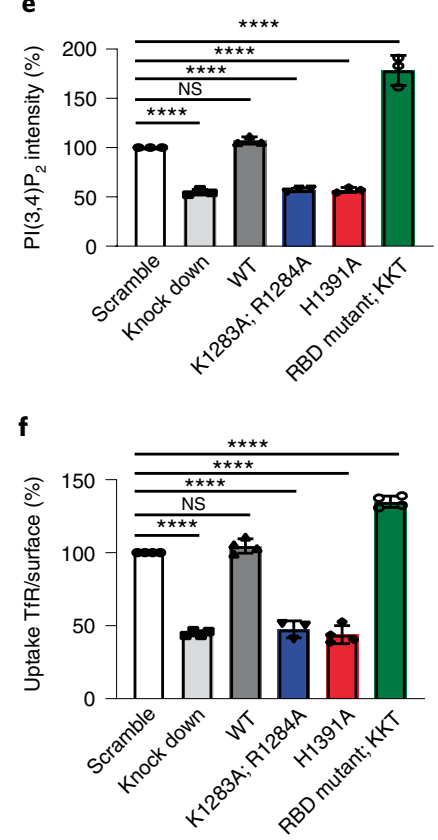

Fig. 5 | Conformational control of PI3KC2 $\alpha$ activity in vitro and at endocytic clathrin-coated pits in living cells. a, Closed conformation of PI3KC2 $\alpha$ based on the docked model in Fig. 4. Only the $\mathrm{N}$ terminus of k $\alpha 12$ (red) is defined. Regions identified as inhibitory contacts by HDX-MS (linker of k $\mathbf{7}$ and $k \alpha 8, \beta 1$ of the RBD, tip of RBD $\alpha 1$ ) are shown with black ribbons. Close-up view of closed contacts I and II with key residues indicated. $\mathbf{b}$, Open conformation of PI3KC2 $\alpha$ based on the crystal structure of PI3KC2 $\alpha^{\Delta \mathrm{N}+\Delta \mathrm{C}-\mathrm{C} 2} \cdot \mathrm{k} \alpha 12$ (green) is completely folded and stabilizes a short helical segment of the activation loop (yellow) that is only defined in the open state. Close-up view indicating crucial interactions between k 12 and the activation loop. c, In vitro PI $(3,4) \mathrm{P}_{2}$-synthesizing activity of PI3KC2 $\alpha$ carrying mutations that disrupt key interactions stabilizing the open or closed states. Deletion of k $\alpha 12$ or the $\mathrm{H} 1391 \mathrm{~A}$ mutation disrupt the open state and cause inactivation of PI3KC2 $\alpha$. The KKT mutation ( ${ }^{1303} \mathrm{EKP}^{1305}$ to ${ }^{1303} \mathrm{KKT}^{1305}$; that is, closed contact I) or mutations in the RBD (K426A, W458A, D462A, D463A; that is, in closed contact II) disrupt the closed state and cause PI3KC2 $\alpha$ hyperactivation. A deletion mutant lacking the C-terminal PX-C2 domains serves as a control. Data represent mean $\pm \mathrm{s}$.d. from $n=3$ experiments. One-way ANOVA with Tukey's multiple comparisons, ${ }^{* *} P=0.0012 ;{ }^{* * *} P=0.0002 ;{ }^{* * * *} P<0.0001$. d,e, Conformational control of local $\mathrm{PI}(3,4) \mathrm{P}_{2}$ synthesis mediated by $\mathrm{PI} 3 \mathrm{KC} 2 \alpha$ at endocytic clathrin-coated pits in living cells. d, High-magnification close-up views of PI3KC2 $\alpha$-depleted Cos7 cells re-expressing siRNA-resistant variants of eGFP-PI3KC2 $\alpha$ WT or mutants (green) stained for PI $(3,4) P_{2}$ (red) and analyzed by total internal reflection fluorescence microscopy. Examples of colocalization of $\mathrm{PI} 3 \mathrm{KC} 2 \alpha$ with $\mathrm{PI}(3,4) \mathrm{P}_{2}$ are highlighted by white arrows. Scale bar, $50 \mu \mathrm{m}$. e, Quantified $\mathrm{PI}(3,4) \mathrm{P}_{2}$ levels at endocytic clathrin-coated pits

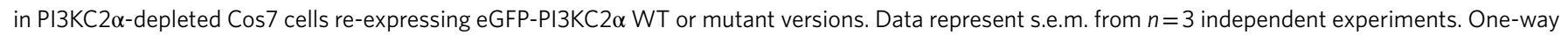
ANOVA with Tukey's multiple comparisons, NS, not significant; ${ }^{* * * *} P<0.0001$. $\mathbf{f}$, Ratio of internalized ( $10 \mathrm{~min}, 37^{\circ} \mathrm{C}$ ) to surface ( $45 \mathrm{~min}, 4^{\circ} \mathrm{C}$ ) transferrin (TfR) in PI3KC2 $\alpha$-depleted Cos7 cells re-expressing eGFP-PI3KC2 $\alpha$ WT or mutant versions. Data represent s.e.m. from $n=4$ independent experiments. One-way ANOVA with multiple Tukey's comparisons, NS, significant; **** $P<0.0001$. 


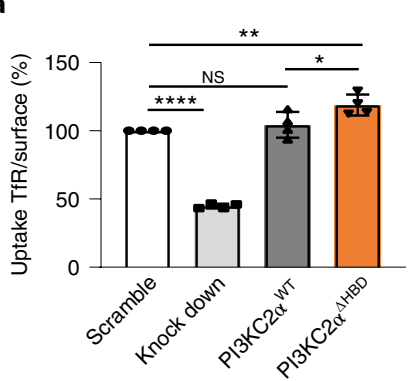

C
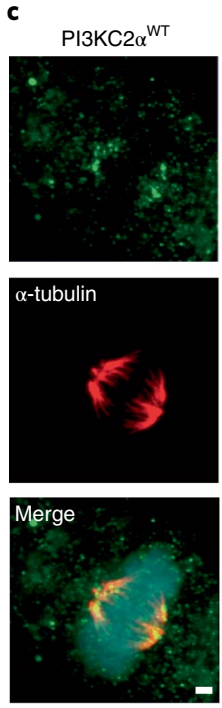

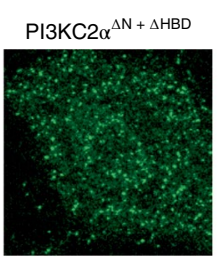

$\alpha$-tubulin
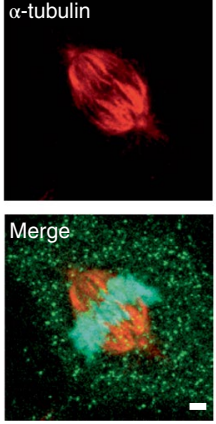

b

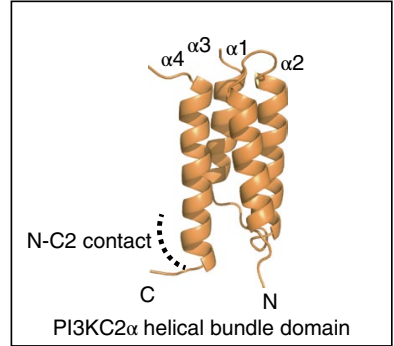

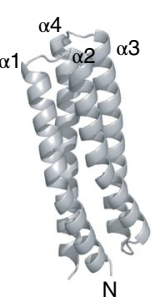

Crk FAT domain

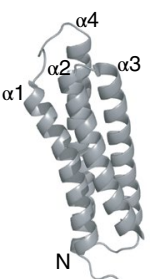

$\alpha$-catenin

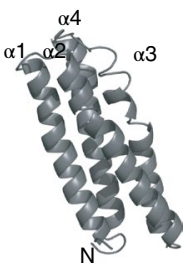

Vinculin

d

PI3KC2 $\alpha^{\text {HBD }}$

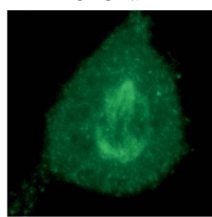

$\alpha$-tubulin
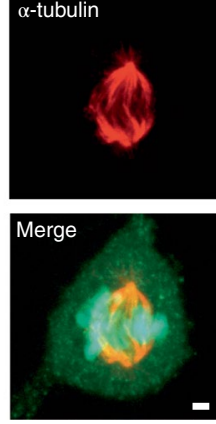

d
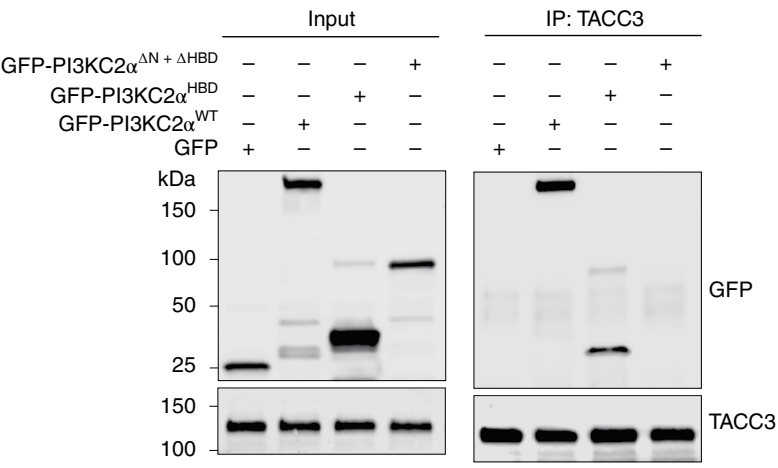

e
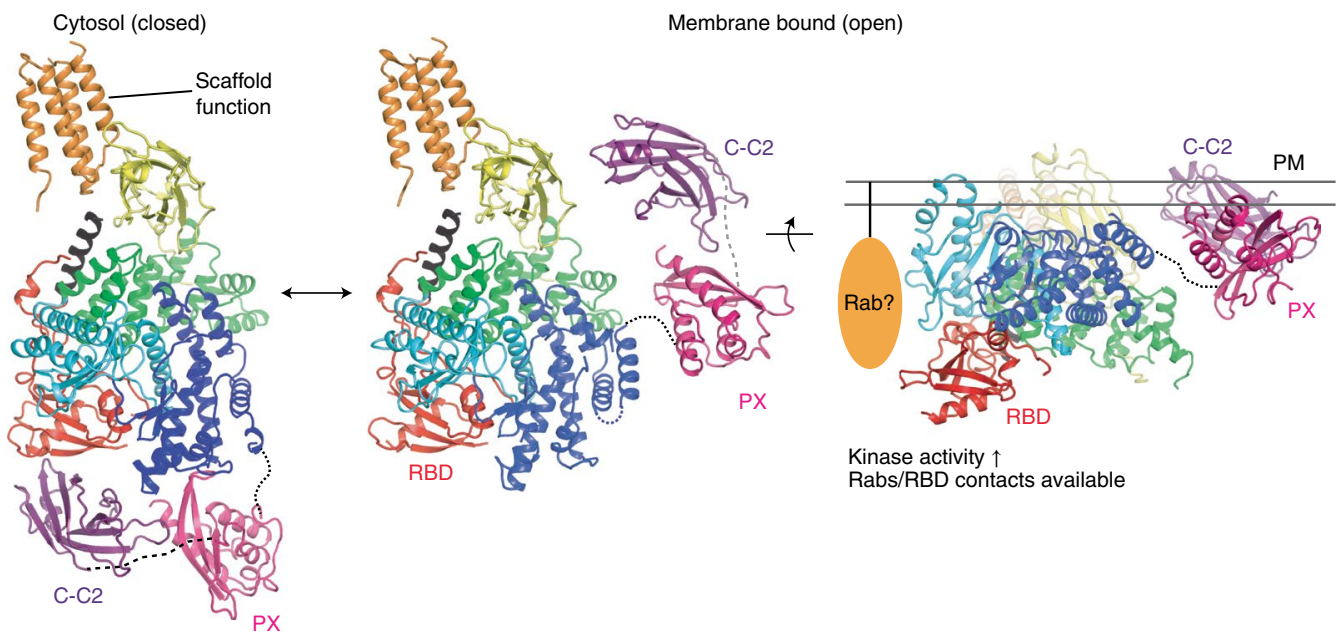

Kinase activity $\uparrow$

Rabs/RBD contacts available

Fig. 6 | Structural basis of the scaffolding function of PI3KC2 $\alpha$ and model for PI3KC2 $\alpha$ activation at membranes. a-c, The unique HBD underlies the scaffold function of PI3KC2 $\alpha . \mathbf{a}$, Bar diagrams representing the ratio of internalized $\left(10 \mathrm{~min}, 37^{\circ} \mathrm{C}\right)$ to surface $\left(45 \mathrm{~min}, 4^{\circ} \mathrm{C}\right)$ transferrin in $\mathrm{PI} 3 \mathrm{KC} 2 \alpha^{\mathrm{WT}}$ or $\mathrm{PI} 3 \mathrm{KC} 2 \alpha^{\triangle \mathrm{HBD}}$ expressing Cos7 cells depleted of the endogenous PI3KC2 $\alpha$ enzyme. The HBD domain is dispensable for clathrin-mediated endocytosis. Data represent s.e.m. from $n=4$ independent experiments, one-way ANOVA with Tukey's multiple comparisons, NS, not significant; ${ }^{*} P=0.0262 ; * * P=0.0046$; ${ }^{* * * *} P<0.0001$. b, Structure of PI3KC2 $\alpha \mathrm{HBD}$ and its closest structural relatives, that is, focal-adhesion targeting (FAT) domain of Crk-associated substrate (Cas) and the F-actin binding domains of vinculin and $\alpha$-catenin. Homology search and ranking was conducted using the DALI server (PDB $3 T 6 G, Z=9.6$, r.m.s.d. $=2.2 \AA$; PDB $6 N R 7, Z=8.5$, r.m.s.d. $=2.0 \AA$, PDB $4 I G G, Z=8.5$, r.m.s.d. $=2.1 \AA$ ). The identified homologs share functional features by serving as scaffolds for proteinprotein interactions. c, The HBD targets PI3KC2 $\alpha$ to the mitotic spindle. Confocal images of fixed metaphase-arrested HeLa cells expressing eGFP-PI3KC2 $\alpha^{\mathrm{WT}}$, PI3KC2 $\alpha^{\triangle N+\triangle H B D}$ or PI3KC2 $\alpha^{H B D}$ immunolabelled for $\alpha$-tubulin (red) (representative of three independent experiments). eGFP-PI3KC2 $\alpha^{W T}$ or PI3KC2 $\alpha^{H B D}$ localize to the mitotic spindle, whereas a mutant lacking the HBD (PI3KC2 $\left.\alpha^{\triangle \mathrm{N}+\Delta H B D}\right)$ displays a diffuse cytosolic localization. DAPI, cyan; PI3KC2 $\alpha$, green and $\alpha$-tubulin, red. Scale bars, $5 \mu \mathrm{m}$. d, TACC3 binds to the HBD of PI3KC2 $\alpha$. Coimmunoprecipitation of endogenous TACC3 and eGFP-PI3KC2 $\alpha$ HBD in metaphase-arrested HEK293T cells expressing WT or mutant versions of eGFP-PI3KC2 $\alpha$, or eGFP. Endogenous TACC3 was immunoprecipitated using anti-TACC3 antibodies (IP, immunoprecipitation) and bound proteins were detected using anti-GFP antibodies. WCL, whole cell lysate. Representative data from three independent experiments are shown. e, Model for PI3KC2 $\alpha$ activation at endocytic membranes. A PI $(4,5) \mathrm{P}_{2}$-induced large-scale conformational rearrangement of the enzyme causes the displacement of the distal C2 domain from the RBD and is likely facilitated by complex formation of the RBD with a Rab protein. The concomitant association of the disordered N-terminal region of PI3KC2 $\alpha$ with clathrin has been omitted for clarity. 
The class III Vps34 complex binds to membranes via the tips of two arms that is three of its four subunits: one contact is formed by the catalytic Vps34 subunit and the Vps15 myristoylation site, the other one involves the Vps30/Beclin 1 BARA domain?

The unique mechanism of membrane binding and activation of $\mathrm{PI} 3 \mathrm{KC} 2 \alpha$ is not only interesting from a mechanistic viewpoint, but also bears important implications for our understanding of class II PI3K biology. Our structural data predict that the conformational activation and, thereby, the catalytic function of PI $3 \mathrm{KC} 2 \alpha$ is triggered by multiple coincident signals, most notably, the membrane association of its PX and C2 domains ${ }^{30,37}$. The exquisite lipid-binding specificity of these domains for $\mathrm{PI}(4,5) \mathrm{P}_{2}$ thereby limits $\mathrm{PI} 3 \mathrm{KC} 2 \alpha$ activity to nanoscale sites enriched in $\mathrm{PI}(4,5) \mathrm{P}_{2}$, providing a structural explanation for the observed spatiotemporal restriction of $\mathrm{PI} 3 \mathrm{KC} 2 \alpha$-mediated synthesis of $\mathrm{PI}(3,4) \mathrm{P}_{2}$ or $\mathrm{PI}(3) \mathrm{P}$ at late-stage endocytic pits $\mathrm{s}^{15,33,41}$ and the base of primary cilia ${ }^{13,42}$. Our structural and biochemical data further predict that the conformational activation of PI3KC2 $\alpha$, and likely other class II PI3K family members, further requires or is facilitated by complex formation of the RBD with an endocytic Rab protein ${ }^{12,13}$. Rab association would aid displacement of the distal C2 domain from the RBD and, thereby, act as a third coincidence determinant, in addition to clathrin ${ }^{15,39}$ and $\mathrm{PI}(4,5) \mathrm{P}_{2}\left(\right.$ ref. $\left.^{30}\right)$. The structure-based mechanism for the activation of PI3KC2 $\alpha$ at membranes described here therefore predicts that the multiple physiological functions of $\mathrm{PI} 3 \mathrm{KC} 2 \alpha$, for example in endocytic receptor internalization and recycling ${ }^{12,15,16,33}$, VEGF-driven angiogenesis ${ }^{19}$ and viral replication ${ }^{21,22}$, result from and are defined by the coincident interaction of $\mathrm{PI} 3 \mathrm{KC} 2 \alpha$ with $\mathrm{PI}(4,5) \mathrm{P}_{2}$ and different Rab proteins that steer its catalytic activity to distinct nanoscale sites. Identifying the respective Rab protein underlying these activities will be key to our understanding of the physiological functions of PI3KC2 $\alpha$ in cell physiology and disease.

Additionally to providing insights into the mechanism of PI3KC2 $\alpha$ activation and function, our structural analysis of PI3KC2 $\alpha$ in complex with nonselective PI3K inhibitors will undoubtedly serve as a door-opener for rational development of isoform-selective PI3KC2 $\alpha$ inhibitors and other class II PI3K family members that may provide new therapeutic avenues for the treatment of important human diseases such as thrombosis ${ }^{28}$, viral infection $^{21,22}$, diabetes ${ }^{29}$ or cancer ${ }^{1,14}$. Finally, we provide a structural basis for the scaffolding function of PI $3 \mathrm{KC} 2 \alpha$ at the mitotic spindle that involves the association of its unique HBD with the microtubule-associated kinetochore protein TACC3 (Fig. 6). These structural insights will be of relevance to develop new therapeutics to fight cancer and cancer metastasis ${ }^{14,26}$.

\section{Online content}

Any methods, additional references, Nature Research reporting summaries, source data, extended data, supplementary information, acknowledgements, peer review information; details of author contributions and competing interests; and statements of data and code availability are available at https://doi.org/10.1038/ s41594-022-00730-w.

Received: 13 July 2021; Accepted: 21 January 2022; Published online: 7 March 2022

\section{References}

1. Bilanges, B., Posor, Y. \& Vanhaesebroeck, B. PI3K isoforms in cell signalling and vesicle trafficking. Nat. Rev. Mol. Cell Biol. 20, 515-534 (2019).

2. Toker, A. \& Cantley, L. C. Signalling through the lipid products of phosphoinositide-3-OH kinase. Nature 387, 673-676 (1997).

3. Wymann, M. P. \& Schneiter, R. Lipid signalling in disease. Nat. Rev. Mol. Cell Biol. 9, 162-176 (2008).

4. Burke, J. E. Structural basis for regulation of phosphoinositide kinases and their involvement in human disease. Mol. Cell 71, 653-673 (2018).

5. Pacold, M. E. et al. Crystal structure and functional analysis of Ras binding to its effector phosphoinositide 3-kinase gamma. Cell 103, 931-943 (2000).
6. Walker, E. H., Perisic, O., Ried, C., Stephens, L. \& Williams, R. L. Structural insights into phosphoinositide 3-kinase catalysis and signalling. Nature 402, 313-320 (1999).

7. Rostislavleva, K. et al. Structure and flexibility of the endosomal Vps34 complex reveals the basis of its function on membranes. Science $\mathbf{3 5 0}$, aac7365 (2015).

8. Stjepanovic, G., Baskaran, S., Lin, M. G. \& Hurley, J. H. Vps34 kinase domain dynamics regulate the autophagic PI 3-kinase complex. Mol. Cell 67, 528-534 e3 (2017)

9. Braccini, L. et al. PI3K-C2gamma is a Rab5 effector selectively controlling endosomal Akt2 activation downstream of insulin signalling. Nat. Commun. 6, 7400 (2015)

10. Marat, A. L. et al. mTORC1 activity repression by late endosomal phosphatidylinositol 3,4-bisphosphate. Science 356, 968-972 (2017).

11. Boukhalfa, A. et al. PI3KC2alpha-dependent and VPS34-independent generation of PI3P controls primary cilium-mediated autophagy in response to shear stress. Nat. Commun. 11, 294 (2020).

12. Campa, C. C. et al. Rab11 activity and PtdIns(3)P turnover removes recycling cargo from endosomes. Nat. Chem. Biol. 14, 801-810 (2018).

13. Franco, I. et al. PI3K class II alpha controls spatially restricted endosomal PtdIns3P and Rab11 activation to promote primary cilium function. Dev. Cell 28, 647-658 (2014).

14. Gulluni, F., De Santis, M. C., Margaria, J. P., Martini, M. \& Hirsch, E. Class II PI3K functions in cell biology and disease. Trends Cell Biol. 29, 339-359 (2019).

15. Posor, Y. et al. Spatiotemporal control of endocytosis by phosphatidylinositol3,4-bisphosphate. Nature 499, 233-237 (2013).

16. Wang, H. et al. Phosphatidylinositol 3,4-bisphosphate synthesis and turnover are spatially segregated in the endocytic pathway. J. Biol. Chem. 295, 1091-1104 (2020).

17. Aki, S., Yoshioka, K., Takuwa, N. \& Takuwa, Y. TGFbeta receptor endocytosis and Smad signaling require synaptojanin1, PI3K-C2alpha-, and INPP4B-mediated phosphoinositide conversions. Mol. Biol. Cell 31, 360-372 (2020).

18. Biswas, K. et al. Essential role of class II phosphatidylinositol-3-kinaseC2alpha in sphingosine 1-phosphate receptor-1-mediated signaling and migration in endothelial cells. J. Biol. Chem. 288, 2325-2339 (2013).

19. Yoshioka, K. et al. Endothelial PI3K-C2alpha, a class II PI3K, has an essential role in angiogenesis and vascular barrier function. Nat. Med. 18, 1560-1569 (2012)

20. Islam, S. et al. Class II phosphatidylinositol 3-kinase alpha and beta isoforms are required for vascular smooth muscle Rho activation, contraction and blood pressure regulation in mice. J. Physiol. Sci. 70, 18 (2020).

21. Abere, B. et al. Kaposi's sarcoma-associated herpesvirus nonstructural membrane protein pK15 recruits the class II phosphatidylinositol 3-kinase PI3K-C2alpha to activate productive viral replication. J. Virol. 92, e00544 (2018).

22. Polachek, W. S. et al. High-throughput small interfering RNA screening identifies phosphatidylinositol 3-kinase class ii alpha as important for production of human cytomegalovirus virions. J. Virol. 90, 8360-8371 (2016).

23. Mountford, J. K. et al. The class II PI 3-kinase, PI3KC2alpha, links platelet internal membrane structure to shear-dependent adhesive function. Nat. Commun. 6, 6535 (2015)

24. Valet, C. et al. Essential role of class II PI3K-C2alpha in platelet membrane morphology. Blood 126, 1128-1137 (2015).

25. Tiosano, D. et al. Mutations in PIK3C2A cause syndromic short stature, skeletal abnormalities, and cataracts associated with ciliary dysfunction. PLoS Genet. 15, e1008088 (2019).

26. Gulluni, F. et al. Mitotic spindle assembly and genomic stability in breast cancer require PI3K-C2alpha scaffolding function. Cancer Cell 32, 444-459 e7 (2017).

27. Young, L. N., Goerdeler, F. \& Hurley, J. H. Structural pathway for allosteric activation of the autophagic PI 3-kinase complex I. Proc. Natl Acad. Sci. USA 116, 21508-21513 (2019).

28. Selvadurai, M. V. et al. Disrupting the platelet internal membrane via PI3KC2alpha inhibition impairs thrombosis independently of canonical platelet activation. Sci. Transl. Med. 12, eaar8430 (2020).

29. Alliouachene, S. et al. Inactivation of class II PI3K-C2alpha induces leptin resistance, age-dependent insulin resistance and obesity in male mice. Diabetologia 59, 1503-1512 (2016).

30. Wang, H. et al. Autoregulation of class ii alpha PI3K activity by its lipid-binding PX-C2 domain module. Mol. Cell 71, 343-351 e4 (2018).

31. Miller, S. et al. Shaping development of autophagy inhibitors with the structure of the Lipid Kinase Vps34. Science 327, 1638-1642 (2010).

32. Siempelkamp, B. D., Rathinaswamy, M. K., Jenkins, M. L. \& Burke, J. E. Molecular mechanism of activation of class IA phosphoinositide 3-kinases (PI3Ks) by membrane-localized HRas. J. Biol. Chem. 292, 12256-12266 (2017).

33. Schoneberg, J. et al. Lipid-mediated PX-BAR domain recruitment couples local membrane constriction to endocytic vesicle fission. Nat. Commun. 8 , 15873 (2017). 
34. Miller, M. S. et al. Structural basis of nSH2 regulation and lipid binding in PI3Kalpha. Oncotarget 5, 5198-5208 (2014).

35. Wymann, M. P. \& Schultz, C. The chemical biology of phosphoinositide 3-kinases. Chem. Bio. Chem. 13, 2022-2035 (2012).

36. Yang, H. et al. mTOR kinase structure, mechanism and regulation. Nature 497, 217-223 (2013)

37. Chen, K. E., Tillu, V. A., Chandra, M. \& Collins, B. M. Molecular basis for membrane recruitment by the PX and C2 domains of class II phosphoinositide 3-kinase-C2alpha. Structure 26, 1612-1625 e4 (2018),

38. Karaca, E., Melquiond, A. S., de Vries, S. J., Kastritis, P. L. \& Bonvin, A. M. Building macromolecular assemblies by information-driven docking: introducing the HADDOCK multibody docking server. Mol. Cell Proteom. 9, 1784-1794 (2010)

39. Gaidarov, I., Smith, M. E., Domin, J. \& Keen, J. H. The class II phosphoinositide 3-kinase C2alpha is activated by clathrin and regulates clathrin-mediated membrane trafficking. Mol. Cell 7 , 443-449 (2001).

40. Hon, W. C., Berndt, A. \& Williams, R. L. Regulation of lipid binding underlies the activation mechanism of class IA PI3-kinases. Oncogene 31 3655-3666 (2012).
41. He, K. et al. Dynamics of phosphoinositide conversion in clathrin-mediated endocytic traffic. Nature 552, 410-414 (2017).

42. Phua, S. C. et al. Dynamic remodeling of membrane composition drives cell cycle through primary cilia excision. Cell 168, 264-+ (2017).

Publisher's note Springer Nature remains neutral with regard to jurisdictional claims in published maps and institutional affiliations.

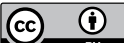

Open Access This article is licensed under a Creative Commons

Attribution 4.0 International License, which permits use, sharing, adaptation, distribution and reproduction in any medium or format, as long as you give appropriate credit to the original author(s) and the source, provide a link to the Creative Commons license, and indicate if changes were made. The images or other third party material in this article are included in the article's Creative Commons license, unless indicated otherwise in a credit line to the material. If material is not included in the article's Creative Commons license and your intended use is not permitted by statutory regulation or exceeds the permitted use, you will need to obtain permission directly from the copyright holder. To view a copy of this license, visit http://creativecommons. org/licenses/by/4.0/.

(c) The Author(s) 2022 


\section{Methods}

Oligonucleotides. Oligonucleotide sequences used in this study are listed in Supplementary Table 1 .

Cell lines. HeLa, human embryonic kidney 293T (HEK293T) and Cos7 cells were obtained from ATCC and cultured in DMEM with $4.5 \mathrm{gl}^{-1}$ glucose (Lonza) containing $10 \%$ heat-inactivated FBS, $100 \mathrm{U} \mathrm{ml}^{-1}$ penicillin and $100 \mu \mathrm{g} \mathrm{ml}^{-1}$ streptomycin (Gibco). Cells were routinely tested for and devoid of mycoplasma contamination.

Cloning and mutagenesis. Complementary DNA encoding mouse PI3KC2 $\alpha$ was synthesized from total RNA extracted from mouse brain using a gene specific primer (5'TAGATACGTTGCCGCAGTCAGCTG3') (Supplementary Table 1) according to the SuperScript III protocol. For baculovirus-mediated expression in insect cells, cDNA encoding mouse PI3KC2 $\alpha^{\Delta \mathrm{N}}$ (amino acids 377-1686), PI3KC2 $\alpha^{\Delta \mathrm{N}+\Delta \mathrm{C}-\mathrm{C} 2}$ (amino acids $\left.377-1,539\right)$ and PI3KC2 $\alpha^{\Delta \mathrm{N}+\Delta \mathrm{PX}-\mathrm{C} 2}$ (amino acids $377-1,400)$ was amplified by PCR and cloned into pFL10His via KasI/XbaI restriction sites. Mutations were introduced by site-directed mutagenesis using PCR. Crystallization construct I, PI3KC $2 \alpha^{\Delta \mathrm{N}+\Delta \mathrm{C}-\mathrm{C} 2}$ contains a re-engineered internal loop with amino acids 533-544 being replaced by the amino acid sequence GSGS. In PI3KC2 $\alpha^{\text {core }}$, the HBD (amino acids 550-665) was replaced by the sequence SGAGSGA. GST-PX (amino acids 1409-1539), GST-PX-C2 (amino acids 1,409-1,686) and GST-distal C2 (amino acids 1,561-1,686) were cloned into pGEX-4T-1 using BamHI and NotI sites for expression in Escherichia coli. The siRNA-resistant enhanced green fluorescent protein- (eGFP-)PI3KC2 $\alpha$ mutant was generated by site-directed mutagenesis PCR based on siRNA-resistant eGFP-PI3KC2 $\alpha$ used in our earlier studies ${ }^{15,30}$.

Protein expression and purification. $\mathrm{His}_{10}$-tagged $\mathrm{PI} 3 \mathrm{KC} 2 \alpha^{\Delta \mathrm{N}}, \mathrm{PI} 3 \mathrm{KC}^{\Delta \mathrm{N}+\Delta \mathrm{C}-\mathrm{C} 2}$ $\mathrm{PI} 3 \mathrm{KC} 2 \alpha^{\text {core }}$ and related mutants were expressed in Sf21 insect cells, using SF900-II serum-free media (ThermoFisher). In brief, $S f 21$ cells $(800 \mathrm{ml})$ grown to a density of $1.5-2 \times 10^{6}$ cells per $\mathrm{ml}$ were infected with $8 \mathrm{ml}$ amplified baculovirus encoding the desired construct. Cells were collected when the viability was less than $90 \%$. Cell pellets were stored frozen at $-20^{\circ} \mathrm{C}$ until purification. For purification, cell pellets from each $200 \mathrm{ml}$ of culture were resuspended in $35 \mathrm{ml}$ of lysis buffer (50 mM Tris pH 7.2, $300 \mathrm{mM} \mathrm{NaCl}, 10 \mathrm{mM}$ imidazole, $1 \mathrm{mM}$ dithiothreitol (DTT), $0.5 \%$ Triton X-100, 1 tablet per $50 \mathrm{ml}$ of protease inhibitor cocktail), sonicated for $1 \mathrm{~min}$ ( $1 \mathrm{~s}$ pulse on, $5 \mathrm{~s}$ pulse off) and centrifuged for $20 \mathrm{~min}$ at $87,000 \mathrm{~g}$. Then $50 \mathrm{ml}$ of supernatant were incubated with $0.5 \mathrm{ml}$ Nickel NTA beads (Sigma Inc.) on a rotating wheel for $1 \mathrm{~h}$. Beads were collected in an open column, washed with $20 \mathrm{ml}$ of lysis buffer, then with $30 \mathrm{ml}$ of wash buffer $(50 \mathrm{mM}$ Tris $\mathrm{pH} 7.5,300 \mathrm{mM}$ $\mathrm{NaCl}, 20 \mathrm{mM}$ imidazole, $1 \mathrm{mM}$ DTT). Protein was eluted with $8 \mathrm{ml}$ of elution buffer ( $20 \mathrm{mM}$ Tris pH 7.5, $300 \mathrm{mM} \mathrm{NaCl}, 300 \mathrm{mM}$ imidazole, $5 \mathrm{mM}$ DTT). The His $\mathrm{m}_{10}$-tag was released by tobacco etch virus ( $10 \mathrm{mg}$ of protein per $0.25 \mathrm{mg}$ of tobacco etch virus) cleavage overnight, while dialyzing against size-exclusion chromatography (SEC) buffer (20 mM Tris pH 7.5, $300 \mathrm{mM} \mathrm{NaCl}, 5 \mathrm{mM} \mathrm{DTT})$ at $4{ }^{\circ} \mathrm{C}$. Proteins were purified on a Superdex 200 gel filtration column at $4^{\circ} \mathrm{C}$ with SEC buffer (20 mM Tris pH 7.5, $300 \mathrm{mM} \mathrm{NaCl}, 5 \mathrm{mM}$ DTT). Proteins were concentrated to about $2.4 \mathrm{mg} \mathrm{ml}^{-1}$ for PI3KC2 $\alpha^{\Delta \mathrm{N}}, 2.5 \mathrm{mg} \mathrm{ml}^{-1}$ for PI3KC2 $\alpha^{\Delta \mathrm{N}+\Delta \mathrm{C}-\mathrm{C} 2}, 5 \mathrm{mg} \mathrm{ml}^{-1}$ for PI3KC2 $\alpha^{\text {core }}$ and $1-2 \mathrm{mg} \mathrm{ml}^{-1}$ for other mutants. All proteins were flash frozen in liquid nitrogen and stored at $-80^{\circ} \mathrm{C}$.

Crystallization of PI3KC2 $\alpha^{\Delta \mathrm{N}+\Delta \mathrm{C}-\mathrm{C} 2}$ and PI3KC2 $\alpha^{\text {core }}$. To obtain PI3KC2 $\alpha^{\Delta \mathrm{N}+\Delta \mathrm{C}-\mathrm{C} 2}$ crystals, $>1,000$ conditions were screened using a Crystal Gryphon robot setup (Art Robbins) with $200 \mathrm{nl}$ of protein solution $\left(2.5 \mathrm{mg} \mathrm{ml}^{-1}\right)$ and $200 \mathrm{nl}$ of screen solution in 96-well sitting-drop plates. Initial crystals were observed with a 50\% water-diluted kit from Molecular Dimensions Morpheus E10 and E11, which originally contains $0.12 \mathrm{M}$ ethylene glycol mix, $0.1 \mathrm{M}$ Tris/Bicine, $40 \% \mathrm{v} / \mathrm{v}$ ethylene glycol, 20\% w/v polyethylene glycol (PEG) 8,000 (in E10) or 40\% glycerol and $20 \%$ PEG 4,000 (in E11). Optimal crystals were obtained at room temperature by microseeding in 24-well sitting-drop plates in mother liquid containing $0.1 \mathrm{M}$ Tris pH 7.5, 8-9\% PEG 20,000, 10\% ethylene glycol and 10\% formamide (for the protein complex with Torin-2:mother liquid:seed of 1:5:0.5 $\mathrm{ll}$ ). Thin plate-shaped crystals grew into large clusters within $2-3 \mathrm{~d}$. Single crystals were isolated and washed in cryogenic solution (50\% fresh prepared mother liquid, $50 \%$ buffer $(20 \mathrm{mM}$ Tris pH 7.5, $150 \mathrm{mM}$ CsI, $5 \mathrm{mM}$ DTT), supplied with $10 \%$ ethylene glycol). Initial PI3KC2 $\alpha^{\text {core }}$ crystals were obtained using homemade screening buffers containing $0.1 \mathrm{M}$ Tris $\mathrm{pH} 7.5,200 \mathrm{mM} \mathrm{MgSO}_{4}, 10 \%$ PEG 8,000. Concentrated protein sample was filtered with $0.2-\mu \mathrm{m}$ spin filters and final crystals were grown in $0.1 \mathrm{M}$ Tris pH 7.5, 100-200 $\mathrm{mM} \mathrm{MgSO}_{4}, 7-10 \%$ PEG 3,350. Crystals were cryo-protected with mother liquid supplied with $10 \%$ ethylene glycol. For ligand soaking, $2 \mathrm{mM}$ ATP, $1 \mathrm{mM}$ Torin-2, $0.5 \mathrm{mM}$ PIK-90 were prepared in cryoprotection solution and incubated with protein crystals for $30 \mathrm{~min}$. Crystals were mounted in a nylon loop and flash cooled in liquid nitrogen.

Data collection, model building and refinement. Diffraction data were collected at station BL14.1 of BESSY/Helmholtz Center Berlin (HZB). Images were processed with XDSAPP ${ }^{43}$. The PI3KC2 $\alpha^{\Delta \mathrm{N}+\Delta \mathrm{C}-\mathrm{C} 2}$ structure was determined by molecular replacement with the PHENIX suite ${ }^{44}$ using the helical domains and
KDs of PI3K $\gamma(\mathrm{PDB} 1 \mathrm{E} 8 \mathrm{X})$ and the PX domain of PI3KC2 $\alpha(2 I W L)$ as search models. The structure was manually built using COOT and iteratively refined using Refmac ${ }^{45}$ and BUSTER ${ }^{46}$ PI3KC $2 \alpha^{\text {core }}$ structures were determined by molecular replacement with PHENIX using the crystal structure of $\mathrm{PI} 3 \mathrm{KC} 2 \alpha^{\Delta \mathrm{N}+\Delta \mathrm{C}-\mathrm{C} 2}$ as a search model. The structures were manually built using $\mathrm{COOT}^{47}$ and refined with PHENIX and BUSTER. Data collection and structure refinement statistics are summarized in Table 1. Ramachandran statistics in the order of favored, allowed, outliers for each structure are: PI3KC $2 \alpha^{\Delta \mathrm{N}+\mathrm{C}-\mathrm{C} 2}(95.26$, $4.74,0 \%), \mathrm{PI} 3 \mathrm{KC} 2 \alpha^{\text {core }}$ apo $(96.91,2.97,0.12 \%)$, PI3KC $2 \alpha^{\text {core }}$ in complex with ATP-Mg ${ }^{2+}(94.6,5.28,0.12 \%)$, PI3KC2 $\alpha^{\text {core }}$ in complex with Torin-2 $(98.76,1.11$, $0.12 \%)$ and PI3KC $2 \alpha^{\text {core }}$ in complex with PIK-90 $(96.78,3.22,0 \%)$. Structural data were deposited in the Protein Data Bank (PDB) and are available under accession numbers 7BI2, 7BI4, 7BI6 and 7BI9.

\section{Negative stain screening of PI3KC2 $\alpha^{\Delta \mathrm{N}}$ in buffer with different salt} concentrations. Purified PI $3 \mathrm{KC} 2 \alpha^{\Delta \mathrm{N}}$ (roughly $2.4 \mathrm{mg} \mathrm{ml}^{-1}$, in $20 \mathrm{mM}$ Tris- $\mathrm{HCl}$ buffer, with $300 \mathrm{mM} \mathrm{NaCl}$ at $\mathrm{pH}$ 7.4) was diluted to a final concentration of $0.02 \mathrm{mg} \mathrm{ml}^{-1}$ into buffers containing $20 \mathrm{mM}$ Tris- $\mathrm{HCl}$, and various concentrations of $\mathrm{NaCl}$ ( 50 to $300 \mathrm{mM}$ ) at $\mathrm{pH} 7.4$, before being negatively stained with $2 \%(\mathrm{w} / \mathrm{v})$ uranyl formate. Negatively stained images were collected using a Tecnai Spirit BioTwin transmission electron microscope (Thermo Fisher Scientific) at $120 \mathrm{kV}$ at a nominal magnification of $\times 49,000(2.26 \AA$ per pixel) on a Gatan Rio CCD camera $(4,000 \times 4,000)$. The sample diluted in a buffer containing $100 \mathrm{mM} \mathrm{NaCl}$ presented a good particle distribution without showing significant aggregates. A group of images was collected at a defocus around -1.5 to $-3.5 \mu \mathrm{m}$. Contrast transfer function (CTF) estimation was performed with CTFFIND 4.1 (ref. ${ }^{48}$ ). Particles were auto-picked using Gautomatch-0.53 (https://www2.mrc-lmb.cam.ac.uk/ research/locally-developed-software/zhang-software/), and 2D class averages were performed with RELION-2.0 and higher ${ }^{49}$.

Cryo-EM sample preparation of PI3KC2 $\alpha^{\Delta \mathrm{N}}$. Purified PI3KC2 $\alpha^{\Delta \mathrm{N}}\left(0.8 \mathrm{mg} \mathrm{ml}^{-1}\right.$ in $20 \mathrm{mM}$ Tris- $\mathrm{HCl}, 100 \mathrm{mM} \mathrm{NaCl}$ at $\mathrm{pH} 7.4$ ) were used for plunge-freezing. Double-application of $3 \mu \mathrm{l}$ of diluted PI $3 \mathrm{KC} 2 \alpha^{\Delta \mathrm{N}}$ were applied onto freshly plasma-cleaned (NanoClean, model 1070, Fischione Instruments) QUANTIFOIL Holey Au-carbon-R2/2 specimen grids, and vitrified by plunge-freezing into liquid ethane using a Mark IV Vitrobot device (Thermo Fisher Scientific). Cryo-EM specimen prepared with different blotting conditions (blot force, blot time) were screened. Final datasets were collected from the cryo-EM grids with thinner uniform ice thickness and good particle orientation distribution.

Cryo-EM single-particle data collection of PI3KC2 $\alpha^{\Delta \mathrm{N}}$ specimen. The cryo-EM single-particle datasets of PI3KC2 $\alpha^{\Delta \mathrm{N}}$ were collected without and with $-30^{\circ}$ stage-tilting on a Titan Krios cryo-transmission electron microscope (Thermo Fisher Scientific) operated at $300 \mathrm{kV}$ and equipped with a $\mathrm{K} 3$ direct electron detector (Gatan, Inc.) device at a nominal magnification of $\times 105,000$ yielding a pixel size at the specimen of $0.837 \AA$ per pixel in counting mode (Table 2). Videos were collected with EPU, a data collecting automation software package (Thermo Fisher Scientific), with an imaging setting of total exposure equal to 60 electrons over 50 fractions in $3 \mathrm{~s}$ and a defocus range between -1.5 and $-2.8 \mu \mathrm{m}$, at the Max Planck Institute of Biophysics, Frankfurt, Germany.

Cryo-EM image processing. During data collection, all datasets were preprocessed 'on-the-fly' using cryoSPARC live ${ }^{50}$ running video motion correction, CTF estimation and automatic particle picking and stream $2 \mathrm{D}$ classification to estimate particle quality. As the streamed 2D classification showed particle features as expected, more data automated positions were defined. Once data collection was completed, all videos from both datasets were imported into cryoSPARC, processed with patch motion correction and CTF estimation, and auto-picked separately. The auto-picked particles were inspected and extracted with a box size of 260 pixels to perform particle cleaning using several rounds of $2 \mathrm{D}$ classification and $3 \mathrm{D}$ heterogeneous refinement (3D classification, if effective) with $\mathrm{C} 1$ symmetry, accordingly. The most homogenous particle sets after cleaning and separation from both datasets were merged, containing about 1 million particles, and taken to perform 3D homogeneous refinement and further 2D classifications as necessary. $3 \mathrm{D}$ variability was performed to show the heterogeneity of the PI3KC2 $\alpha^{\Delta \mathrm{N}}$ specimen due to its flexibility. Fourier shell correlation (FSC) estimation was done both in cryosparc and Relion. Postprocessing and local resolution was estimated in RELION-3.0, and global directional resolution estimation was performed using 3DFSC (ref. ${ }^{51}$ ). A detailed image processing pipeline is shown in Extended Data Fig. 5. Cryo-EM data were deposited in the PDB and are available under accession number EMD-12191. Original EM micrographs were deposited in the Electron Microscopy Public Image Archive (EMPIAR) (code EMPIAR-10665).

ADP-Glo kinase assay of PI3KC2 $\alpha$. Purified PI3KC2 $\alpha$ variants were prediluted to $0.5 \mathrm{mg} \mathrm{ml}^{-1}$ in SEC buffer used for purification. All variants were subsequently diluted to $20 \mu \mathrm{g} \mathrm{ml}^{-1}$ in kinase buffer ( $5 \mathrm{mM}$ HEPES/KOH, pH 7.2, $25 \mathrm{mM} \mathrm{KCl}$, $2.5 \mathrm{mM} \mathrm{Mg}(\mathrm{OAc})_{2}, 150 \mathrm{mM}$ K-glutamate, $\left.10 \mu \mathrm{M} \mathrm{CaCl}_{2}, 0.2 \% \mathrm{CHAPS}\right)$. Native liver PI or PI 4-phosphate (PI(4)P) were dissolved to a concentration of $400 \mu \mathrm{M}$ with kinase buffer by water-bath sonification and then supplied with $200 \mu \mathrm{M}$ ATP. 
The buffer supplied with $200 \mu \mathrm{M}$ ATP served as a negative control. Reactions were started by mixing $5 \mu \mathrm{l}$ of protein stock with $5 \mu \mathrm{l}$ of substrate solution and incubated for $20 \mathrm{~m}$ at room temperature. The reactions were stopped by adding $10 \mu \mathrm{l}$ of ADP-Glo reagent (Promega). After 40 min of incubation, $20 \mu \mathrm{l}$ of Kinase Detection Reagent were added. After $20 \mathrm{~min}$ incubation, luminescence was read with a TECAN plate reader. IC $_{50}$ measurements of Torin- 2 and PIK-90 were carried out using concentration series of these compounds.

XL-MS analysis of PI3KC2 $\alpha^{\Delta \mathrm{N}}$. Purified PI3KC2 $\alpha^{\Delta \mathrm{N}}$ was buffer exchanged to crosslinking buffer $(20 \mathrm{mM}$ BisTris-propane, $\mathrm{pH} 7.5,300 \mathrm{mM} \mathrm{NaCl})$ by dialysis. $1.2 \mathrm{mg} \mathrm{ml}^{-1} \mathrm{PI} 3 \mathrm{KC} \alpha^{\Delta \mathrm{N}}$ was diluted into $20 \mathrm{mM}$ BisTris-propane, $\mathrm{pH} 7.5$, to final concentration of $0.4 \mathrm{mg} \mathrm{ml}^{-1}$. Crosslinking was performed by adding $0.5 \mu \mathrm{l}$ of disuccinimidyl sulfoxide (DSSO) (stock concentration $50 \mathrm{mM}$ ) to $50 \mu$ l diluted PI3KC2 $\alpha^{\Delta \mathrm{N}}$. The reaction was performed twice for $20 \mathrm{~min}$ each. Samples were quenched by addition of $1 \mathrm{M}$ Tris $\mathrm{pH} 7.5$ (final concentration $20 \mathrm{mM}$ ) for $30 \mathrm{~min}$. Crosslinked PI3KC2 $\alpha^{\Delta \mathrm{N}}$ was separated by SDS-PAGE followed by in-gel tryptic digest. Crosslinked peptides were analyzed using a Thermo Scientific Dionex UltiMate 3000 system combined to an Orbitrap Fusion Lumos mass spectrometer. Cross-link acquisition was performed using a MS2-MS3 method. MS2 spectra were acquired on every selected MS1 precursor whereas MS3 acquisitions were triggered if a unique mass difference of 31.9721 was observed in the MS2 spectrum ${ }^{52}$. Data analysis was conducted using XlinkX standalone ${ }^{52}$ with the following parameters: minimum peptide length of 6; maximal peptide length of 35 ; missed cleavages of 3; fix modification: Cys carbamidomethyl of $57.021 \mathrm{Da}$; variable modification, Met oxidation of $15.995 \mathrm{Da}$; DSSO crosslinker $158.0038 \mathrm{Da}$ (short arm $54.0106 \mathrm{D}$ and long arm $85.9824 \mathrm{Da}$ ); precursor mass tolerance of $10 \mathrm{ppm}$ and fragment mass tolerance of $20 \mathrm{ppm}$. Results were reported at $1 \%$ false discovery rate (FDR) at the level of cross-link spectrum matches (CSM).

Model of PI3KC2 $\alpha^{\Delta \mathrm{N}}$. To build the model with PX and C2 domain, all of identified crosslinked sites between PX and C2 were introduced with 5-15 $\mathrm{A}(\mathrm{C} \beta$ to $\mathrm{C} \beta$ ) distance restraints for docking of PX and C2 by HADDOCK. In total, 24 docked $\mathrm{PX}-\mathrm{C} 2$ structures from six different clusters were generated. The PX-C2 domain together with PI3KC2 $\alpha^{\text {core }}$ were used to fit into the EM map with Chimera. The best fitted structures were validated with the crosslinked sites between PI3KC2 $\alpha^{\text {core }}$ and PX-C2 domain to obtain the final model.

GST pull down assay. GST pull down assays were performed with immobilized GST-fused PX, C2 or PX-C2 of PI3KC2 $\alpha$ as baits. Then $10 \mu \mathrm{g}$ of immobilized GST fusion protein was used to capture $200 \mu \mathrm{g}$ of PI3KC2 $\alpha^{\Delta \mathrm{N}+\Delta \mathrm{PX}-\mathrm{C} 2} \mathrm{WT}$ or $\mathrm{PI} 3 \mathrm{KC} 2 \alpha^{\Delta \mathrm{N}+\Delta \mathrm{PX}-\mathrm{C} 2} \mathrm{RBD}$ mutant in $500 \mu$ l of binding buffer $(20 \mathrm{mM}$ Tris, $\mathrm{pH} 7.5$, $100 \mathrm{mM} \mathrm{NaCl}$ ). Samples were incubated on a rotating wheel for $1 \mathrm{~h}$ at $4{ }^{\circ} \mathrm{C}$, unbound material was removed by washing three times and bound proteins were eluted with $2 \times$ SDS-PAGE sample buffer. Samples were analyzed by SDS-PAGE and Coomassie blue staining.

Plasmid transfections. Cells were seeded and transfected with $50 \mu \mathrm{M}$ siRNA on day 1 using jetPRIME (Polyplus Transfection) according to the manufacturer's instructions by reverse transfection. A second round of knockdowns was performed on day 2. Cells were plated onto a Matrigel (BD Biosciences) -coated cover slides in the morning of day 3 and transfected with eGFP-PI3KC2 $\alpha$ constructs using jetPRIME (Polyplus Transfection) according to the manufacturer's instructions.

\section{Analysis of the localization of eGFP-PI3KC2 $\alpha$ in metaphase-arrested} cells. HeLa cells were synchronized in metaphase using $2 \mathrm{mM}$ thymidine (Sigma-Aldrich) for $20 \mathrm{~h}, 30 \mu \mathrm{M}$ deoxycitidine for $6 \mathrm{~h}$ (Sigma-Aldrich) and $50 \mathrm{ng} \mathrm{ml}^{-1}$ nocodazole (Sigma-Aldrich) for $12 \mathrm{~h}$, followed by $2 \mathrm{~h}$ release in fresh medium in the presence of $20 \mathrm{mM}$ MG132 (Calbiochem). HeLa cells were blocked in interphase by starvation for $16 \mathrm{~h}$. Synchronized cells were transfected with plasmids encoding eGFP-PI3KC2 $\alpha$ WT or mutants. For immunofluorescence, the cells were fixed by ice-cold methanol. HeLa cells were permeabilized with $0.1 \%$ Saponin for $10 \mathrm{~s}$ and then fixed in $2 \%$ paraformaldehyde (PFA) for $5 \mathrm{~min}$. Staining for GFP, $\alpha$-tubulin and 4,6-diamidino-2-phenylindole (DAPI) was performed, and the cells were examined with a Zeiss Observer-Z1 microscope, equipped with the Apotome, Leica TCS-II SP5 or a Leica TSC-II SP8 confocal microscope.

Coimmunoprecipitation of TACC3 and PI3KC2 $\alpha$. HEK293T were transfected with plasmids encoding eGFP-PI3KC $2 \alpha$ WT or mutants, mitotically arrested $24 \mathrm{~h}$ post-transfection and gathered as reported in ref. ${ }^{26}$. The following primary antibodies were used: TACC3 (Rabbit, no. 8069, Cell Signaling) and homemade GFP (Rabbit, polyclonal). Membranes probed with the indicated antibodies were then incubated with HRP-conjugated anti-Rabbit IgG light chain (1:5,000, 211-032-171, Jackson ImmunoResearch) and developed with enhanced chemiluminescence (ECL, BD).

$\mathrm{PI}(3,4) \mathrm{P}_{2}$ detection at the plasma membrane. PI3KC $2 \alpha \mathrm{KD}$ Cos7 cells or $\mathrm{PI} 3 \mathrm{KC} 2 \alpha \mathrm{KD}$ Cos 7 cells re-expressing eGFP-PI3KC2 $\alpha$ WT or mutants were grown on Matrigel (BD Biosciences)-coated eight-well glass bottom $\mu$-slide (ibidi). Cos7 cells were washed with PBS containing $10 \mathrm{mM} \mathrm{MgCl}_{2}$ once and fixed in $2 \%$ PFA with $0.5 \%$ glutaraldehyde for $20 \mathrm{~min}$ at room temperature. Cells were washed three times with PBS and twice with PBS containing $50 \mathrm{mM}$ $\mathrm{NH}_{4} \mathrm{Cl}$. Cells were permeabilized with PBS containing 0.5\% Saponin and $1 \%$ BSA for 30 min. PI(3,4)P2 antibody (Echlon catalog no. Z-P034b) and Goat anti-Mouse IgG $(\mathrm{H}+\mathrm{L})$ AF647 labeled secondary antibody (Thermo Fisher catalog no. A21237) were incubated for 2 and $1 \mathrm{~h}$, respectively, in PBS buffer containing $1 \%$ BSA and $10 \%$ normal goat serum. Cells were analyzed by total internal reflection fluorescence microscopy (Nikon TI Eclipse, 488 and $561 \mathrm{~nm}$ laser, $\times 60$ numerical aperture 1.49 objective and sCMOS Andor mNeo). Plasma membrane $\mathrm{PI}(3,4) \mathrm{P}_{2}$ levels at clathrin-coated pits were quantified using ImageJ software.

Transferrin uptake and surface labeling. Cos7 cells transfected with siRNA and/or PI3KC2 $\alpha$ (WT or mutant)-encoding plasmids or pretreated for $4 \mathrm{~h}$ with $0.1 \%$ dimethylsulfoxide or $20 \mu \mathrm{M}$ PITCOIN1 were starved in serum-free DMEM media for $1 \mathrm{~h}$. For transferrin uptake, cells were incubated with $25 \mathrm{mg} \mathrm{ml}^{-1}$

Alexa647 labeled transferrin (Molecular Probes, Invitrogen) for $10 \mathrm{~min}$ at $37^{\circ} \mathrm{C}$ in a humidity chamber. Cells were washed twice with ice-cold PBS supplied $10 \mathrm{mM}$ $\mathrm{MgCl}_{2}$ and then acid washed twice at pH5.3 (0.2 M sodium acetate, $200 \mathrm{mM}$ sodium chloride) on ice for $2 \mathrm{~min}$ to remove surface-bound transferrin. Cells were then washed twice more with ice-cold PBS containing $10 \mathrm{mM} \mathrm{MgCl}_{2}$ and fixed with $4 \%$ PFA for $45 \mathrm{~min}$ at room temperature. For surface labeling, cells were incubated with $25 \mathrm{mg} \mathrm{ml}^{-1}$ Alexa647 labeled transferrin at $4{ }^{\circ} \mathrm{C}$ for $45 \mathrm{~min}$ and then washed three times with ice-cold PBS $\left(10 \mathrm{mM} \mathrm{MgCl}_{2}\right)$ on ice for $1 \mathrm{~min}$. Cells were fixed with $4 \%$ PFA for $45 \mathrm{~min}$ at room temperature. Transferrin labeling was analyzed using the Nikon Eclipse Ti microscope and ImageJ software. Internalized transferrin per cell was quantified and normalized to the amount of surface-bound transferrin determined in the same experiment as a measure for the efficiency of internalization.

Statistical analysis. All data are presented as mean \pm s.e.m. and were obtained from $\geq 3$ independent experiments with total sample numbers provided in the figure legends. Statistical significance was evaluated by Prism software (GraphPad), using one simple, two-tailed $t$-test with theoretical mean of 100 or one-way analysis of variance (ANOVA) test with Tukey's multiple comparisons. Specific $P$ values are indicated in the legends to figures. Significant differences were marked as ${ }^{\star} P<0.05$, ${ }^{* *} P<0.01,{ }^{* *} P<0.001$ and ${ }^{* * * *} P<0.0001$

Reporting Summary. Further information on research design is available in the Nature Research Reporting Summary linked to this article.

\section{Data availability}

Structural data were deposited in the PDB and are available under accession numbers 7BI2, 7BI4, 7BI6 and 7BI9 (X-ray crystallography) (Table 1) and EMD-12191 (cryo-EM) (Table 2). Original EM micrographs have been deposited in the EMPIAR and are available under the code EMPIAR-10665. All other data are available in the main manuscript, extended data, supplementary materials and in the source data. Materials and reagents are available from the corresponding authors upon request. Source data are provided with this paper.

\section{References}

43. Sparta, K. M., Krug, M., Heinemann, U., Mueller, U. \& Weiss, M. S. Xdsapp20. J. Appl. Crystallogr. 49, 1085-1092 (2016).

44. Liebschner, D. et al. Macromolecular structure determination using X-rays, neutrons and electrons: recent developments in Phenix. Acta Crystallogr. D. Struct. Biol. 75, 861-877 (2019).

45. Murshudov, G. N. et al. REFMAC5 for the refinement of macromolecular crystal structures. Acta Crystallogr. D. Biol. Crystallogr. 67, 355-367 (2011).

46. Bricogne, G. Direct phase determination by entropy maximization and likelihood ranking: status report and perspectives. Acta Crystallogr. D. Biol. Crystallogr. 49, 37-60 (1993)

47. Emsley, P., Lohkamp, B., Scott, W. G. \& Cowtan, K. Features and development of Coot. Acta Crystallogr. D. Biol. Crystallogr. 66, 486-501 (2010)

48. Rohou, A. \& Grigorieff, N. CTFFIND4: fast and accurate defocus estimation from electron micrographs. J. Struct. Biol. 192, 216-221 (2015).

49. Scheres, S. H. RELION: implementation of a Bayesian approach to cryo-EM structure determination. J. Struct. Biol. 180, 519-530 (2012).

50. Punjani, A., Rubinstein, J. L., Fleet, D. J. \& Brubaker, M. A. cryoSPARC: algorithms for rapid unsupervised cryo-EM structure determination. Nat. Methods 14, 290-296 (2017).

51. Tan, Y. Z. et al. Addressing preferred specimen orientation in single-particle cryo-EM through tilting. Nat. Methods 14, 793-796 (2017).

52. Liu, F., Lossl, P., Scheltema, R., Viner, R. \& Heck, A. J. R. Optimized fragmentation schemes and data analysis strategies for proteome-wide cross-link identification. Nat. Commun. 8, 15473 (2017). 


\section{Acknowledgements}

We are indebted to M.C. Wahl (Freie Universität Berlin, Berlin, Germany) for help with insect cell expression, M. Bommer (Max-Delbrück Center, Berlin, Germany) for help with data collection and processing, and D. Löwe and U. Fink (both from FMP Berlin, Germany) for technical assistance. We also thank BESSY/HZB (Berlin, Germany) staff scientists for support during data collection at beamline MX14.1. Supported by in-house funds from the FMP. Y.Z. and M.K. are supported by a Sofja Kovalevskaja Award from the Alexander von Humboldt Foundation. Y.Z. was partially supported by an IMPRES international student scholarship from the Max Planck Society. M.K. is supported by the Heisenberg Program of the Deutsche Forschungsgemeinschaft (grant no. KU 3222/3-1). O.D. acknowledges funding by the Deutsche Forschungsgemeinschaft (grant no. TRR186/A23 to O.D.)

\section{Author contributions}

W.-T.L., O.V., A.V.Z. and Y.R. performed and O.D. supervised X-ray crystallographic studies. Y.Z. and M.K. contributed cryo-EM analyses. H.S. and F.L. together with W.-T.L. carried out XL-MS. F.G., M.C.D.S. and E.H. analyzed PI3KC2a localization at the spindle. W.-T.L. purified recombinant proteins for all assays, contributed all cell biochemical and cell biological experiments and together with Y.R. built the structural models. W.-T.L. and V.H. conceived and supervised the study, and wrote the paper with input from all authors. Correspondence and requests for materials should be addressed to V.H. (haucke@fmp-berlin.de) or W.-T.L. (lo@fmp-berlin.de).

\section{Funding}

Open access funding provided by Leibniz-Forschungsinstitut für Molekulare Pharmakologie im Forschungsverbund Berlin e.V. (FMP)

\section{Competing interests}

The authors declare no competing interests.

\section{Additional information}

Extended data are available for this paper at https://doi.org/10.1038/ s41594-022-00730-w.

Supplementary information The online version contains supplementary material available at https://doi.org/10.1038/s41594-022-00730-w.

Correspondence and requests for materials should be addressed to Wen-Ting Lo or Volker Haucke.

Peer review information Nature Structural and Molecular Biology thanks Roger Williams, Alexander Leitner and Jun-Jie Liu for their contribution to the peer review of this work. Peer reviewer reports are available. Carolina Perdigoto was the primary editor on this article and managed its editorial process and peer review in collaboration with the rest of the editorial team.

Reprints and permissions information is available at www.nature.com/reprints. 
a

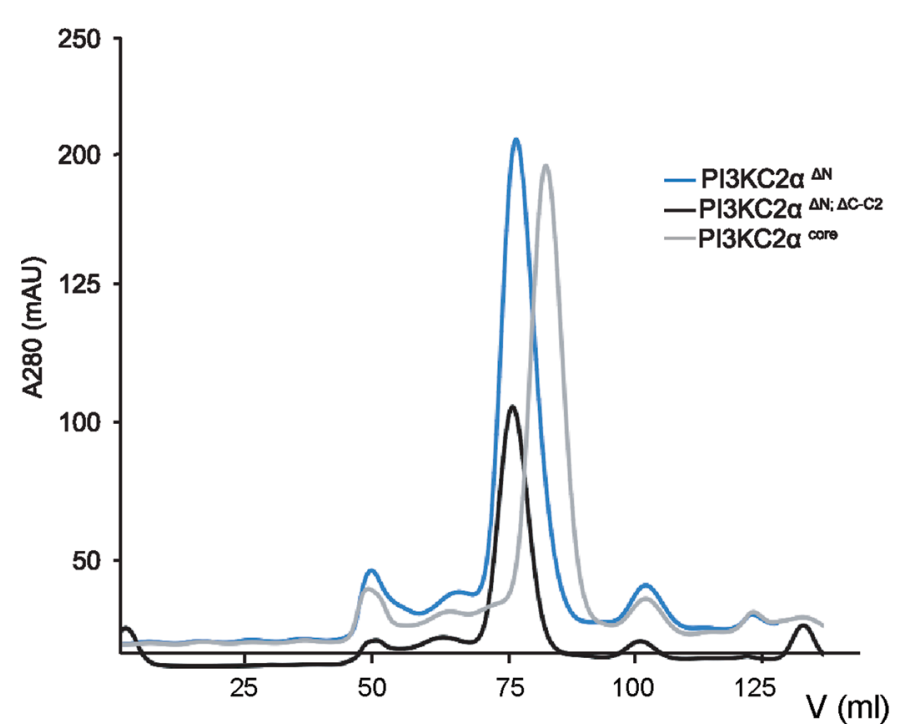

b

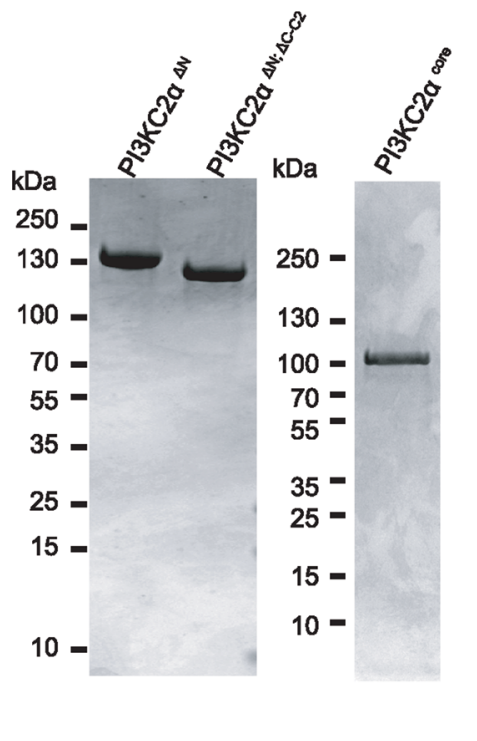

C

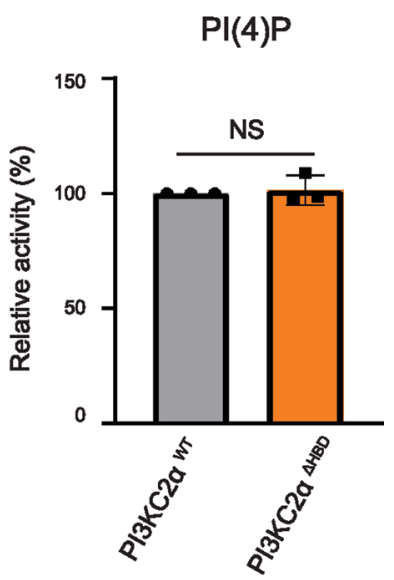

Extended Data Fig. 1 | Purification and lipid kinase activity of PI3KC2 $\alpha^{\Delta \mathrm{N}}$, PI3KC2 $\alpha^{\Delta \mathrm{N}+\Delta \mathrm{C}-\mathrm{C} 2}$, and PI3KC2 $\alpha^{\text {core }}$. (a) Size exclusion chromatography of $\mathrm{PI} 3 \mathrm{KC} 2 \alpha^{\Delta \mathrm{N}}, \mathrm{PI} 3 \mathrm{KC} 2 \alpha^{\Delta \mathrm{N}+\Delta \mathrm{C}-\mathrm{C} 2}$ and PI3KC2 $\alpha^{\text {core }}$. Peak fractions were collected for structural analysis. (b) Coomassie Blue-stained SDS-PAGE of purified $\mathrm{PI} 3 K C 2 \alpha^{\Delta N}, \mathrm{PI} 3 K C 2 \alpha^{\Delta N+\Delta C-C 2}$ and PI3KC2 $\alpha^{\text {core }}$ from (a). (c) In vitro kinase activity of PI3KC2 $\alpha^{W T}$ and PI3KC2 $\alpha^{\Delta H B D}$ using PI(4)P as a substrate. HBD deletion showed no influence on kinase activity. Data represent mean \pm SD from triplicate $(n=3)$ experiments. One sample, 2 tailed $t$-test with hypothetical mean of $100, \mathrm{NS}=$ non-significant. 


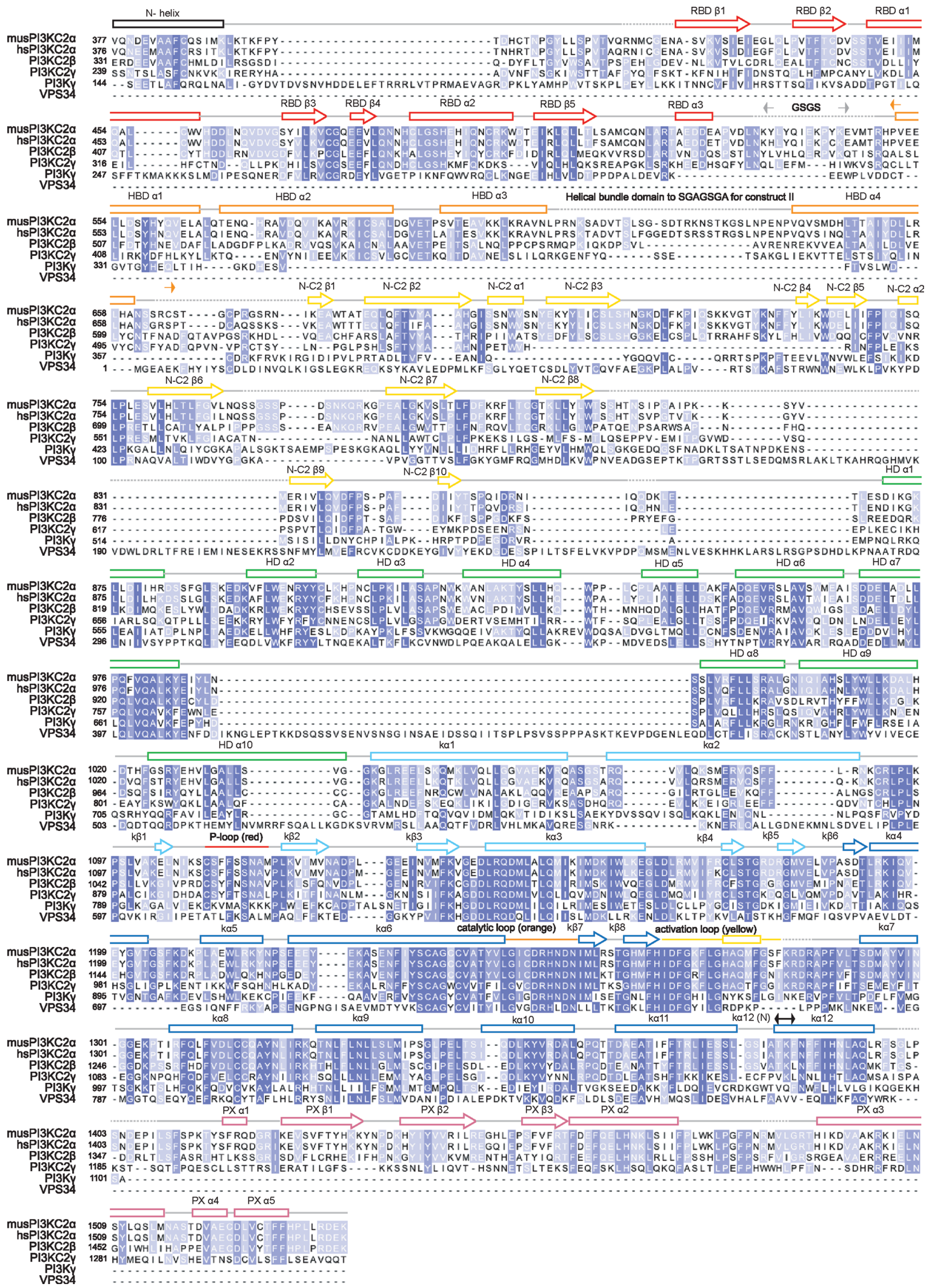

Extended Data Fig. 2 | See next page for caption. 
Extended Data Fig. 2 | Secondary structure of PI3KC2 $\alpha$ and primary sequence alignment with PI3Ks. The sequence corresponding to crystallization construct I (that is musPI3KC2 $\alpha^{\Delta \mathrm{N}+\Delta \mathrm{C}-\mathrm{C} 2}$ ) was used as template to align human PI3KC2 $\alpha$ (hs PI3KC2 $\alpha$, UniProt accession: 000443.2), other human class II PI3K isoforms [PI3KC2 $\beta$ (UniProt accession: 000750.2), PI3KC2 $\gamma$ (UniProt accession: 075747.3)], human class I PI3K (PI3K $\gamma$, UniProt accession: P48736.3), and human class III PI3K (VPS34, UniProt accession: Q8NEB9.1). The dashed lines indicate disordered linker regions. All identified secondary structural elements are color-coded as in Fig. 1a. The light grey arrow indicates the replacement of a short, disordered sequence by GSGS to improve crystallization of PI3KC2 $\alpha^{\Delta \mathrm{N}+\Delta \mathrm{C}-\mathrm{C} 2}$. To generate the $\triangle \mathrm{HBD}$ construct, the HBD was replaced by the sequence SGAGSGA as indicated by the orange arrow. $k \alpha 12(N)$ is indicated by a black arrow. 


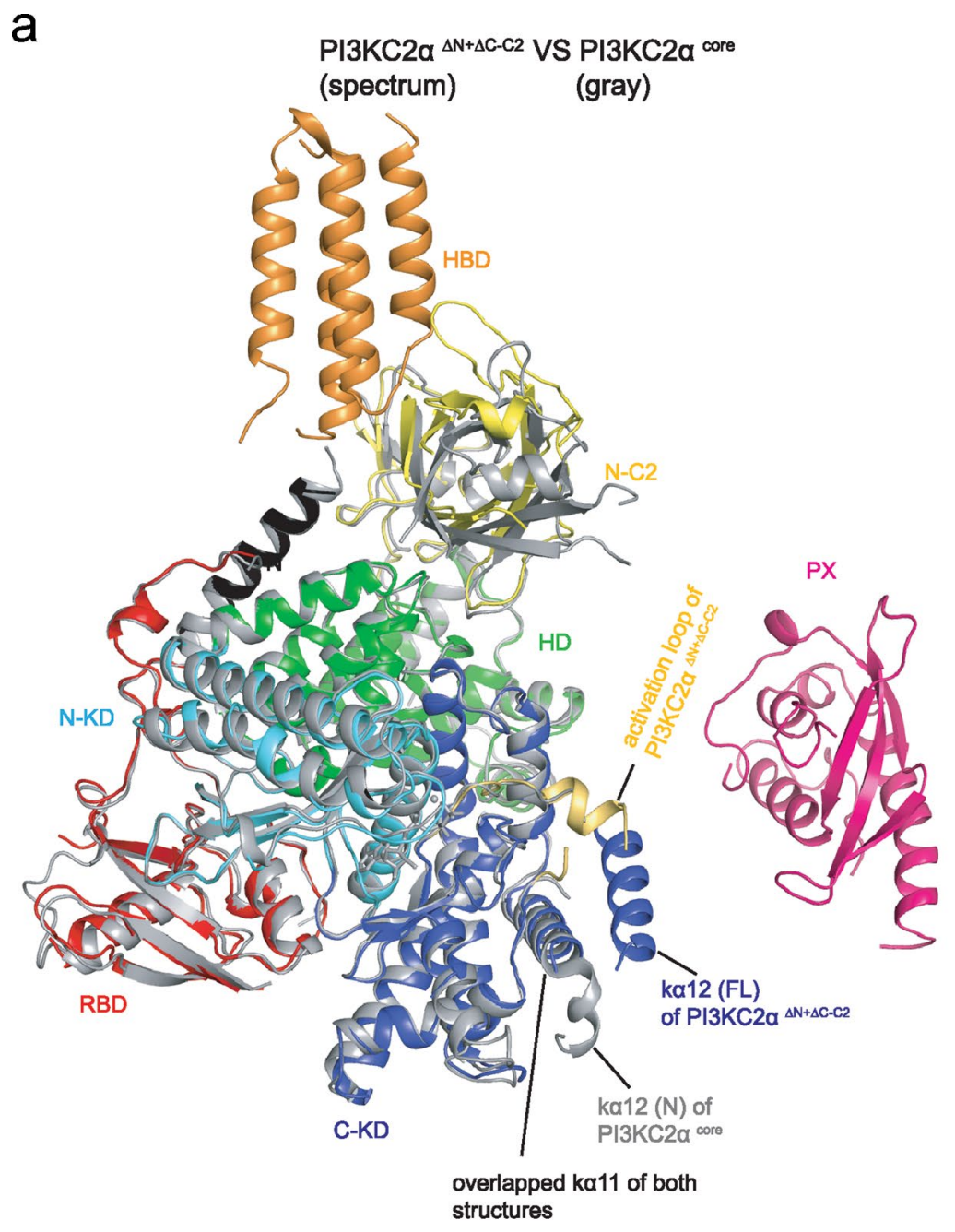

b

\section{C}
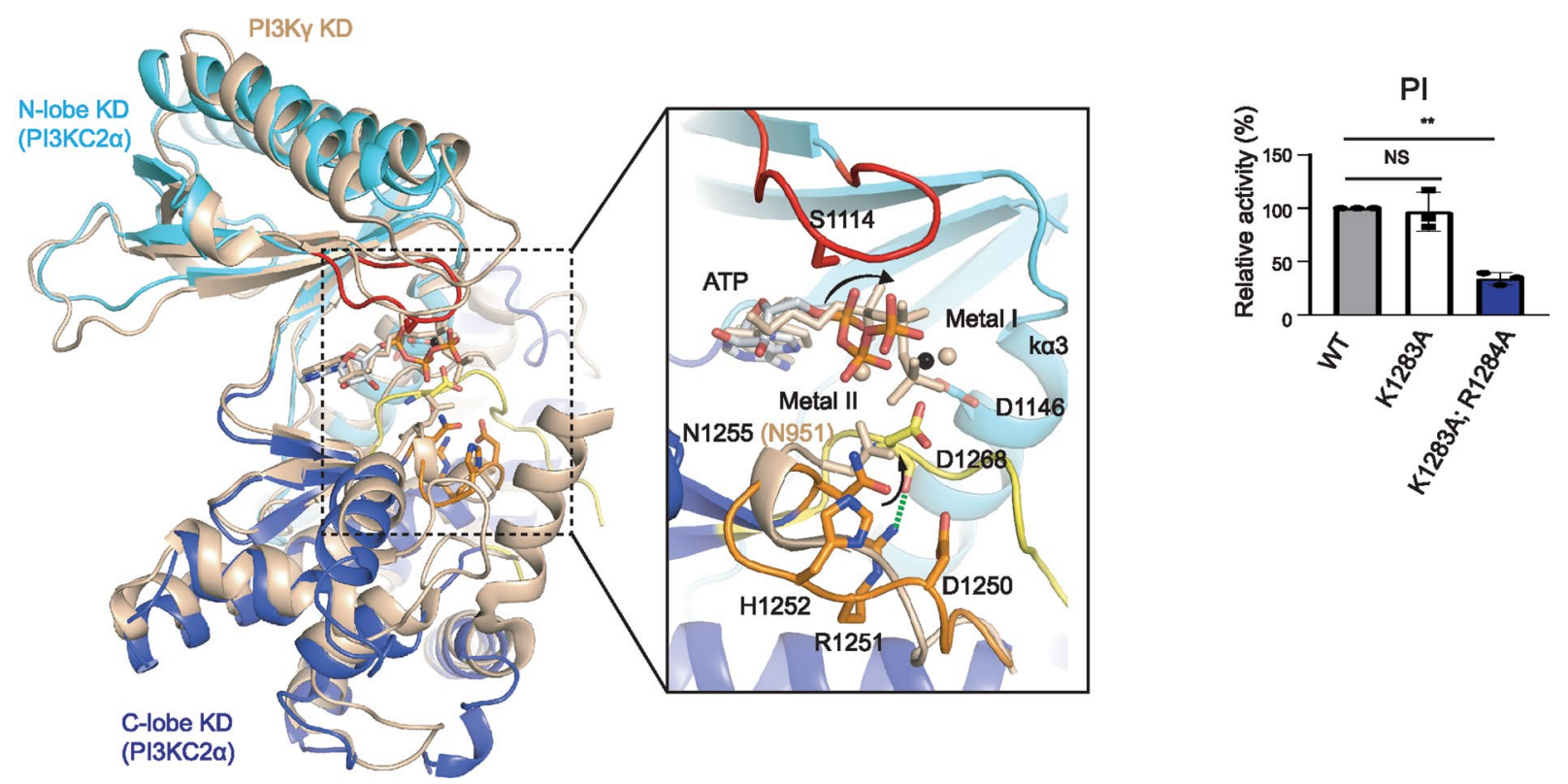

Extended Data Fig. 3 | See next page for caption. 
Extended Data Fig. 3 | Catalytic activity and structure of PI3KC2 $\alpha$. (a) Structural superimposition of PI3KC2 $\alpha^{\Delta \mathrm{N}+\Delta \mathrm{C}-\mathrm{C2}}$ (spectrum) and PI3KC2 $\alpha^{\mathrm{core}}$ (gray). The main distinctive features are the conformations of k $\alpha 12$ and of the activation loop. In the PI3KC2 $\alpha^{\Delta \mathrm{N}+\Delta \mathrm{C}-\mathrm{C} 2}$ structure, a short helical segment within

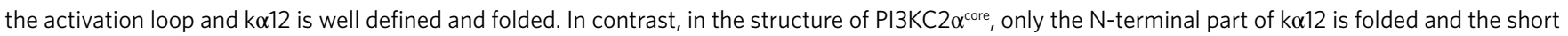
helical segment within the activation loop of PI3KC2 $\alpha$ core is disordered. (b) Structural superimposition of the kinase domains from PI3KC2 $\alpha$ (N-lobe: cyan; C-lobe: blue) and PI3K $\gamma$ (1E8X; wheat). Close-up view of the proposed movement of catalytic loop residues, N1255, and ATP with the appearance of a second metal ion (metal II). Wheat spheres indicate two $\mathrm{Mg}^{2+}$ ions bound to PI3K $\gamma$, black sphere represents the single $\mathrm{Mg}^{2+}$ bound to PI3KC2 $\alpha$. (c) In vitro kinase activity of activation loop mutants with PI as a substrate. The K1283A and R1284A mutations greatly reduce activity. Data represent mean \pm SD from triplicate $(n=3)$ experiments. One sample, 2 tailed t-test with hypothetical mean of $100, N S=$ non-significant; ${ }^{* *} p=0.0025$. 
a<smiles></smiles>

\section{Torin-2}

$\mathrm{PI} 3 \mathrm{KC} 2 \alpha$
IC50 (nM)

64.0 b<smiles>COc1ccc2c(c1OC)N=C(NC(=O)c1cccnc1)N1CCN=C21</smiles>

C

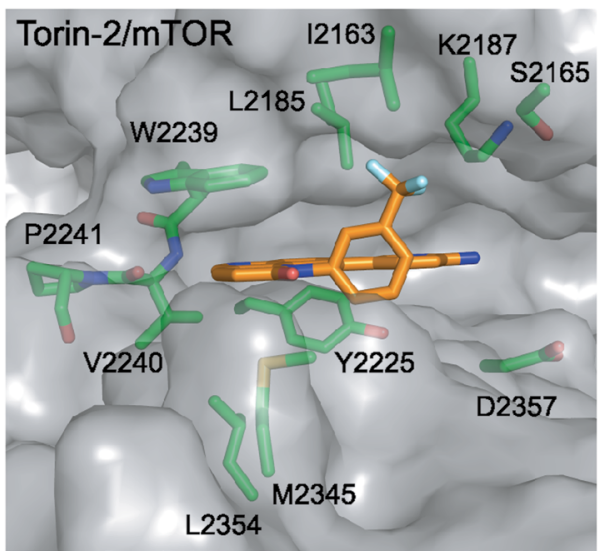

PIK90

PI3KC2a
IC50 (nM)

78.0

d

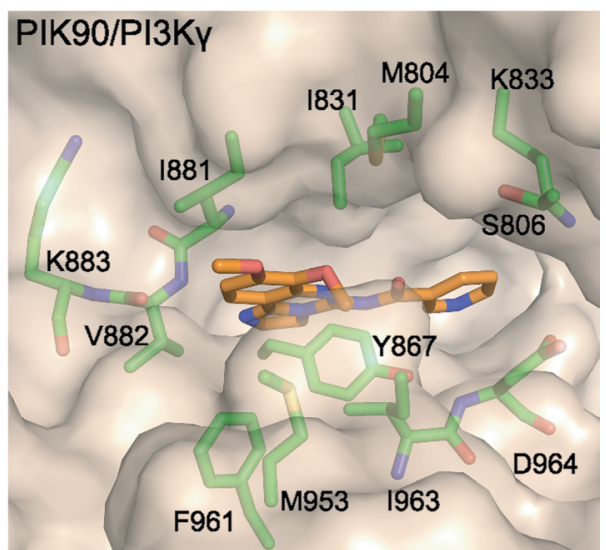

Extended Data Fig. 4 | Pharmacological inhibition of mTOR kinase and PI3K $\gamma$ by Torin-2 and PIK-90. (a,b) 2D structure and IC $\mathrm{C}_{50}$ of Torin-2 (a) and PIK-90 (b) bound to PI3KC2 $\alpha$. (c) Binding pocket of mTOR in association with Torin-2 (pdb: 4JSX). W2239 from the hinge of mTOR kinase contributes a pi-pi interaction with Torin-2. (d) Binding pocket of PI3K $\gamma$ in complex with PIK-90 (pdb: 2CHX). The pyridine ring of PIK-90 binds to a deep cavity in the affinity pocket of $\mathrm{PI} 3 \mathrm{~K} \gamma$. 
a
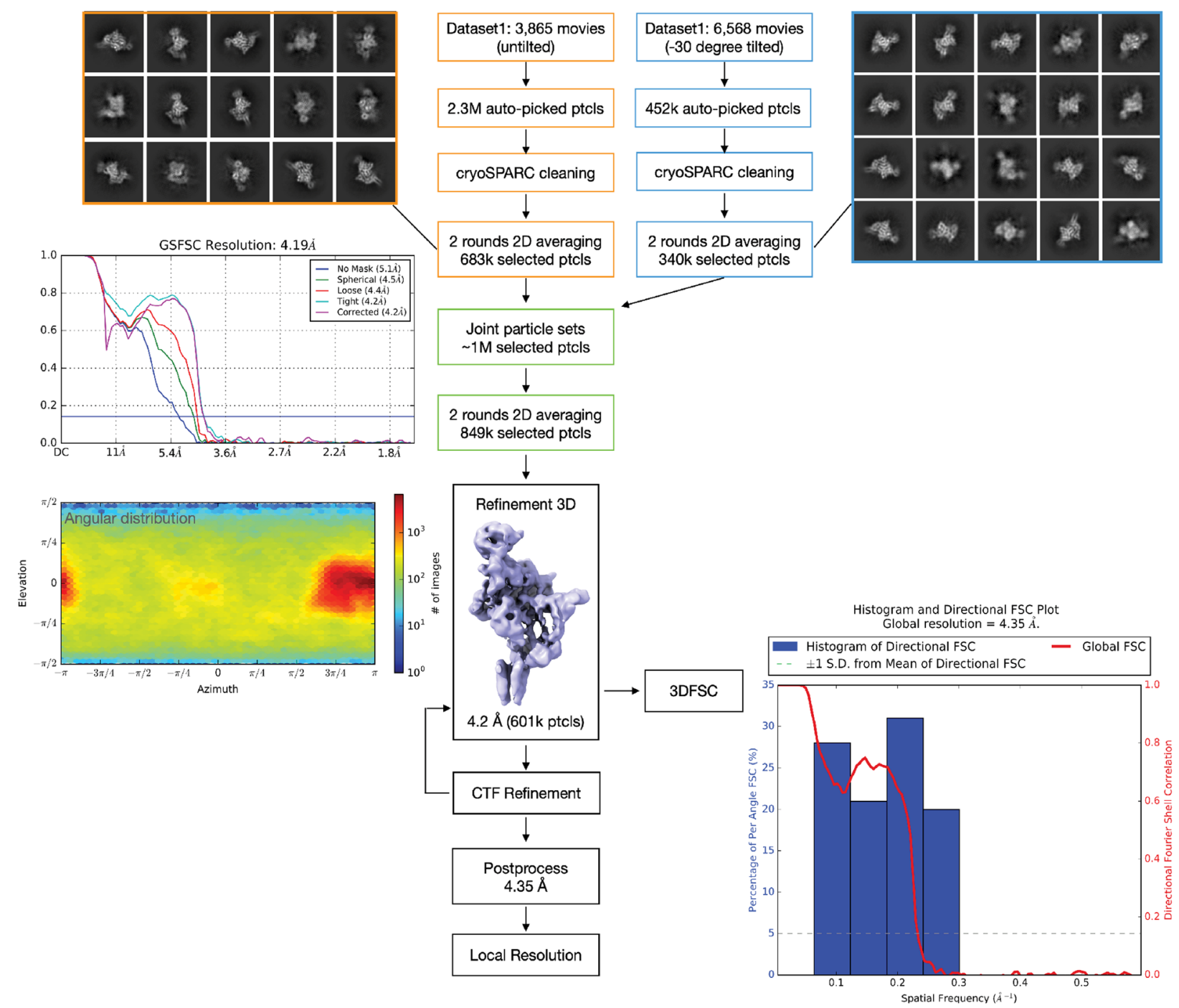

b

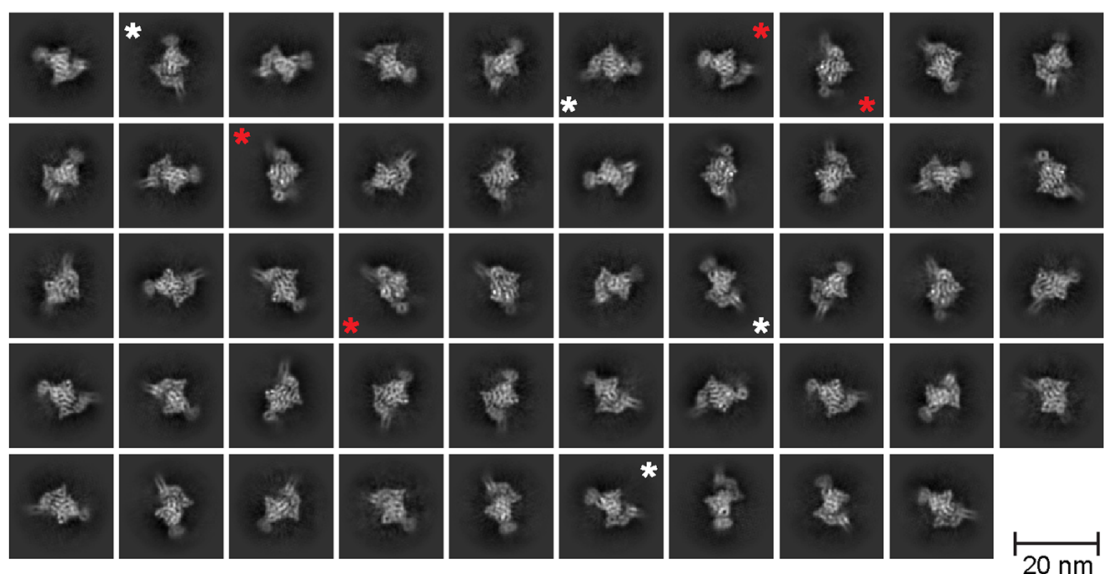

C

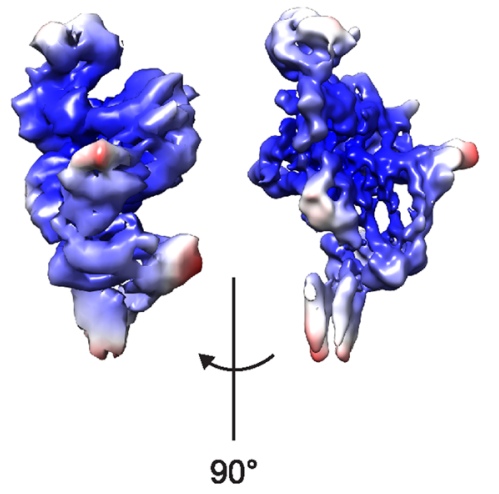

Extended Data Fig. 5 | Cryo-EM structure determination of PI3KC2 $\boldsymbol{\alpha}^{\mathbf{\Lambda N}}$. (a) Cryo-EM image processing workflow. $2.3 \times 10^{6}$ of particles were extracted for 2D classification. $683 \mathrm{k}$ particles were selected after two rounds of 2D classification. $340 \mathrm{k}$ selected particles from $2 \mathrm{D}$ classes based on datasets with 30 tilted angles were joined for a third round of 2D averaging. 601k selected particles were finally used to obtain a 3D reconstruction with a global resolution $4.35 \AA$. The angular distribution is represented as a heat map to indicate the distributed projections of all particles contributing to the consensus map. (b) Representative $2 \mathrm{D}$ classes of PI3KC2 $\alpha^{\Delta \mathrm{N}}$ after 2 rounds of $2 \mathrm{D}$ averaging. (c) Local resolution of the PI3KC2 $\alpha^{\Delta \mathrm{N}} \mathrm{EM}$-map shown as a heat map from $4.04 \AA$ (blue) to $6.71 \AA$ (red) with two different views. The central core region of PI3KC2 $\alpha$ displays a compact helix-rich fold with the highest local resolution. 
a

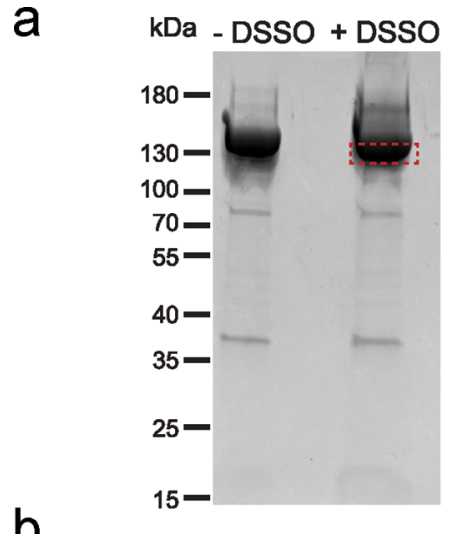

b

cross-linking site:

core/ PX or core/ C2

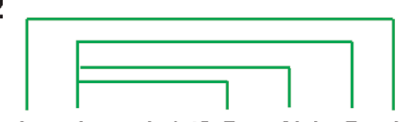

ํㅗำ

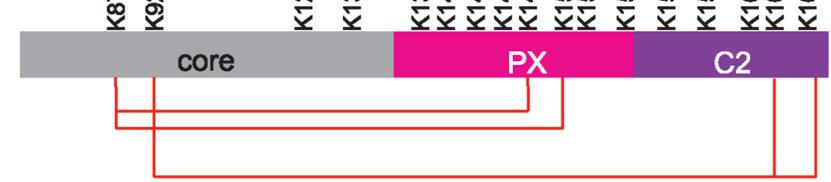

cross-linking site: $\mathrm{PX} / \mathrm{C} 2$

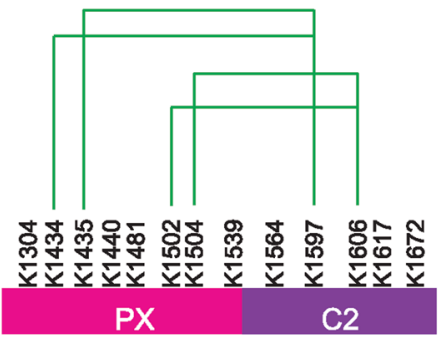

d

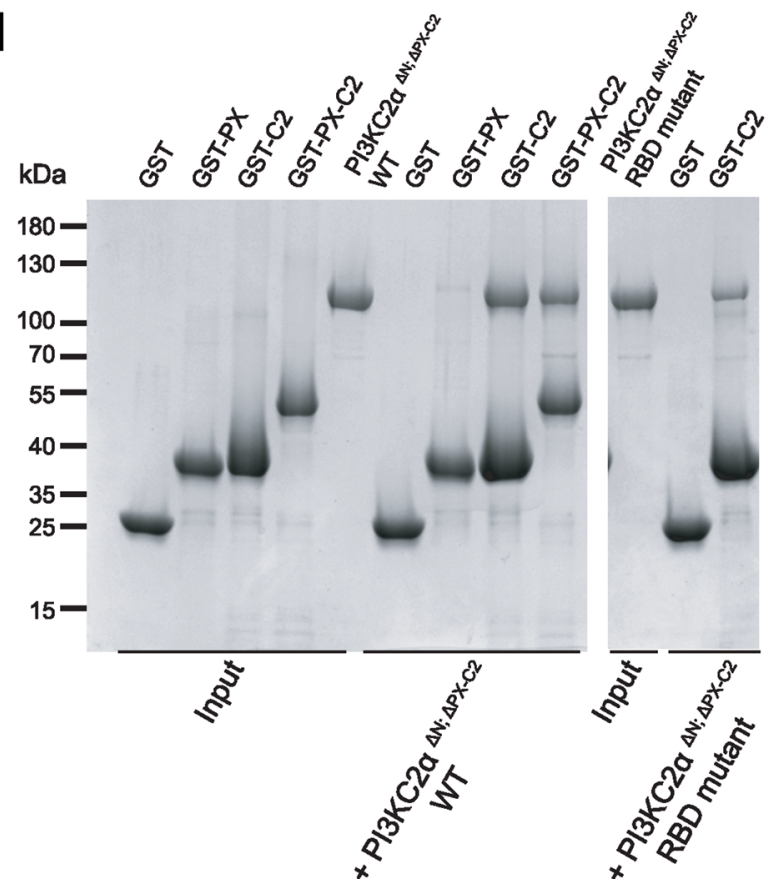

C

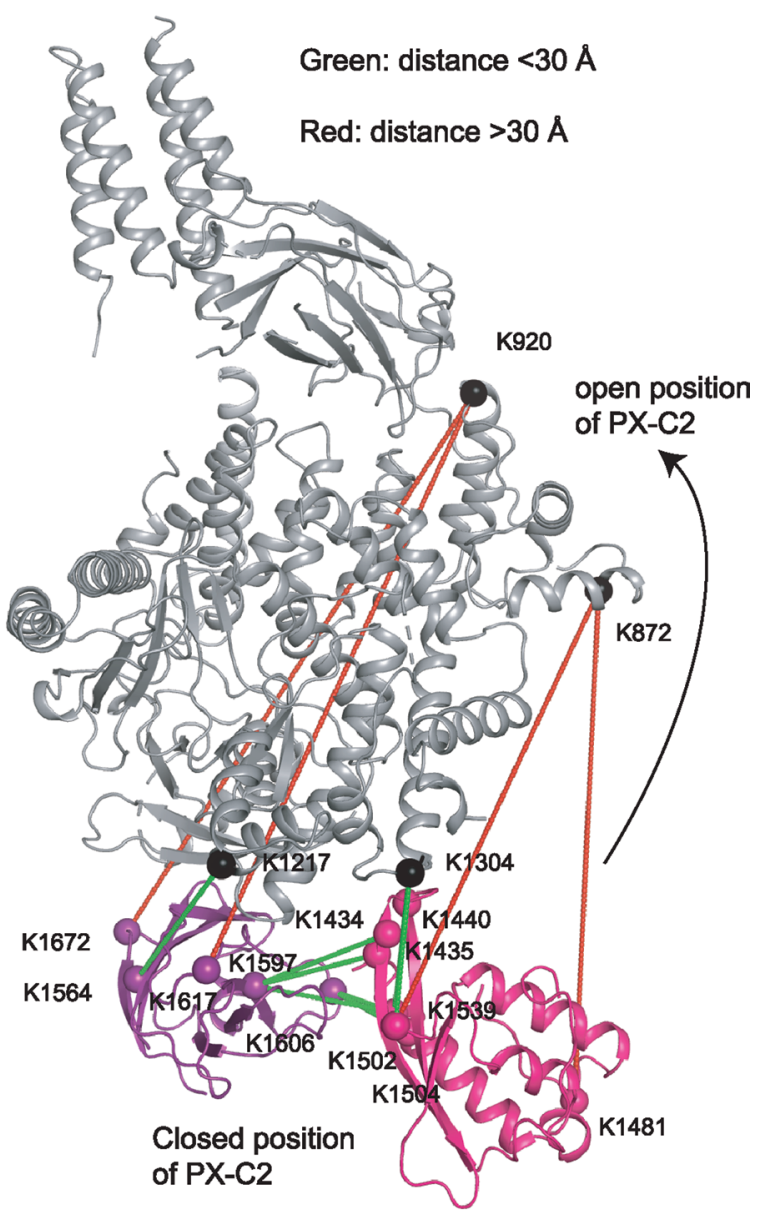

Extended Data Fig. 6 | See next page for caption. 
Extended Data Fig. 6 | Different conformational states of PI3KC2 $\alpha$. (a) PI3KC2 $\alpha^{\Delta N}$ was crosslinked with DSSO chemical crosslinker and separated by $4-15 \%$ gradient SDS-PAGE. The monomeric band of crosslinked PI3KC2 $\alpha^{\Delta \mathrm{N}}$ indicated as dashed red square was cut out for further MS analysis. (b) Identified crosslinked peptides using DSSO as chemical crosslinker. (c) Model of PI3KC2 $\alpha^{\Delta \mathrm{N}}$ based on the cryo-EM map without the PX domain. The PX domain was docked onto the distal C2 domain using restraints imposed by the obtained crosslinks between the PX and distal C2 domain, using the HADDOCK server. The obtained model was subsequently verified manually using all identified crosslinking sites between the kinase core, PX, and distal C2 domains and the C- to $\mathrm{N}$ - terminal connections between these domains. Green line: distance $<30 \AA$; red line; distance $>30 \AA$. Distant crosslinks $(>30 \AA$ ) suggest different conformational states of PI3KC2 $\alpha$. (d) Interaction between the kinase core and lipid binding C2 domains of PI3KC2 $\alpha$. GST pull down assays using GST-PX, GST-C2 or GST-PX-C2 as baits to capture PI3KC2 $\alpha^{\Delta \mathrm{N}+\Delta P X-C 2}$ Wild-type (WT) or RBD mutant (K426A; W458A; D461A; D462A) versions. Samples were analyzed by SDS-PAGE and Coomassie Blue staining and quantified using ImageJ software. Binding of PI3KC2 $\alpha^{\Delta \mathrm{N}+\Delta P X-C 2}$ WT was set to $100 \%$. Data represent SEM from triplicate $(n=3)$ experiments. 
a

\section{$\mathrm{PI} 3 \mathrm{Ky}$}

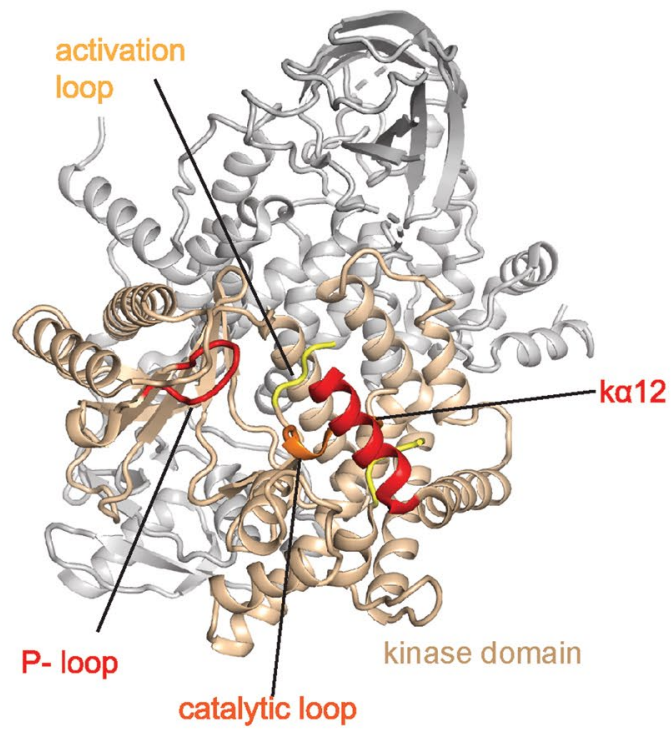

C

\section{$\mathrm{PI} 3 \mathrm{KC} 2 \alpha$}
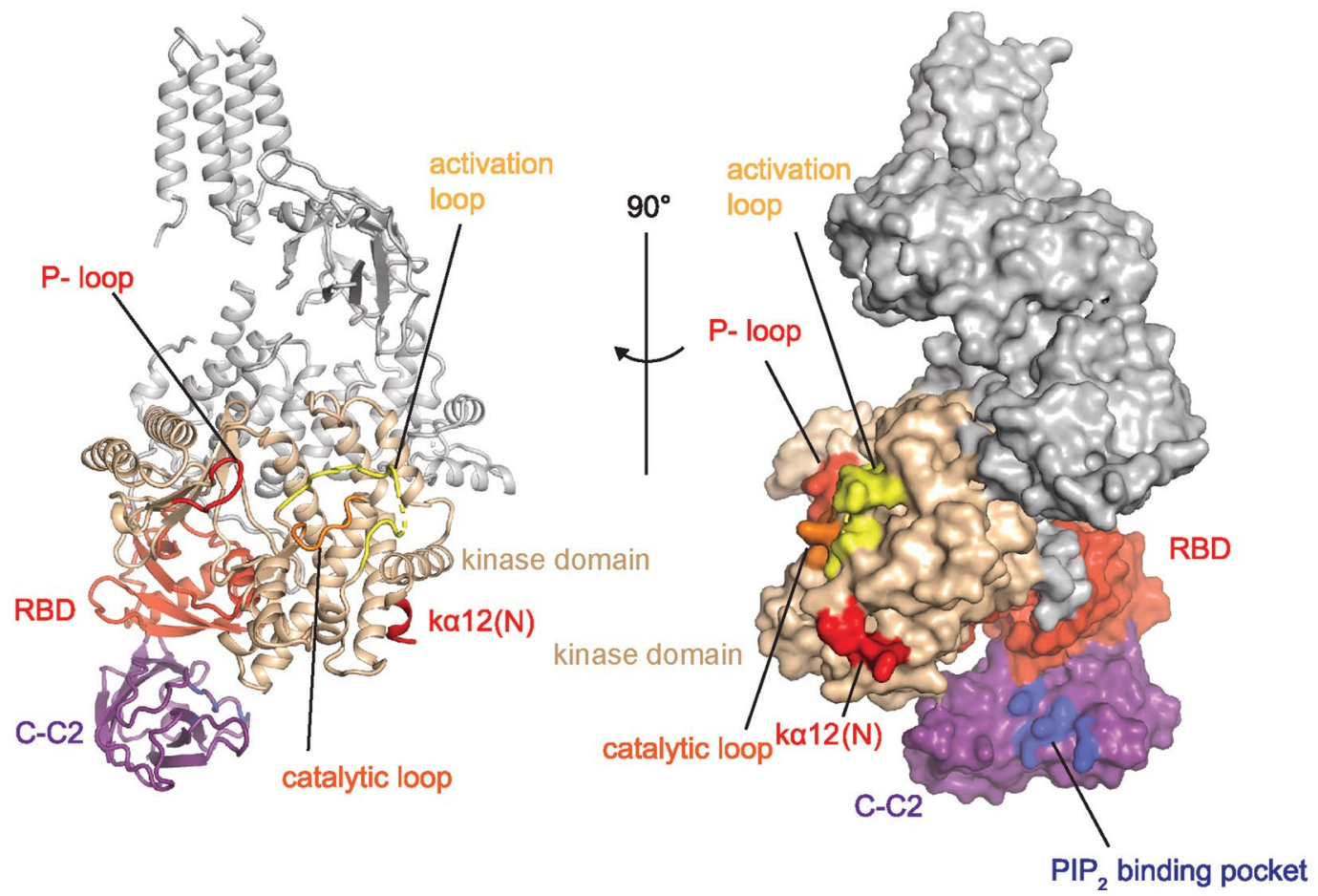

Extended Data Fig. 7 | See next page for caption.

b

\section{VPS34 complex}

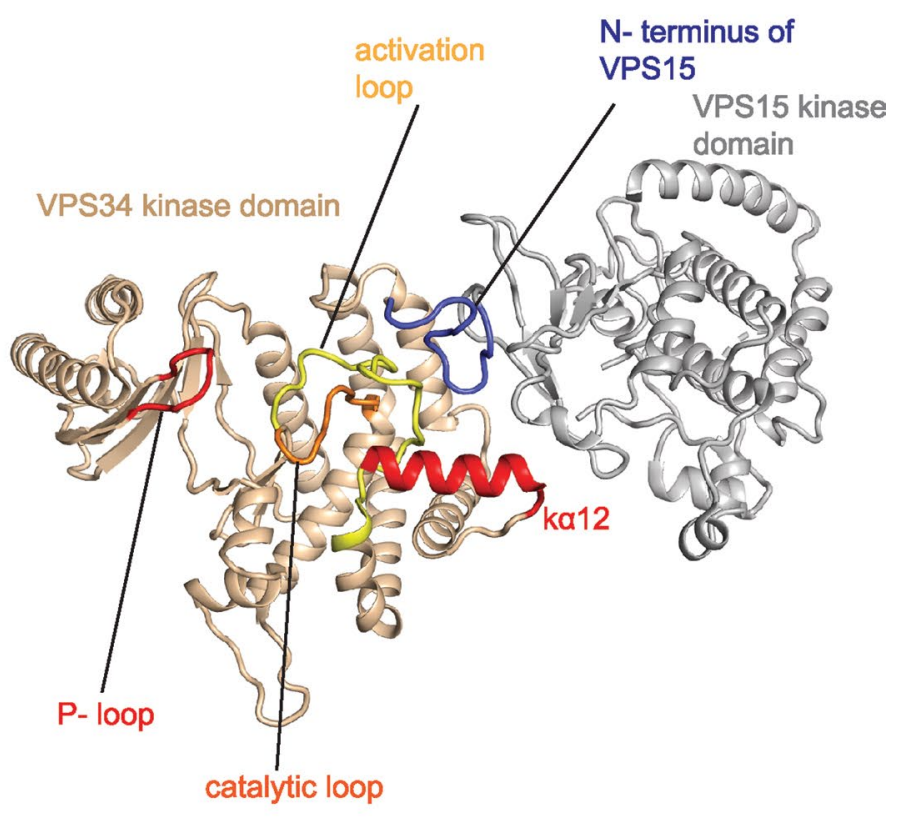


Extended Data Fig. 7 | Comparison of the inhibitory conformations of PI3Ks. (a) Auto-inhibited conformation of PI3K $\gamma$ (pdb: IE8X). k $\alpha 12$ (red) bends inward towards the catalytic pocket. (b) Auto-inhibited conformation of yeast Vps34/Vps15 in complex II (pdb: 5DFZ). The activation loop of VPS34 (yellow) is locked by k $\alpha 12$ (red) and the N-terminus of VPS15 (blue). (c) Auto-inhibited conformation of PI3KC2 $\alpha^{\Delta \mathrm{N}}$ as ribbon and surface representation. The distal C2 domain binds to the RBD. The yellow and orange regions represent the catalytic pocket and the activation loop, where lipid substrates bind. The $\mathrm{PI}(4,5) \mathrm{P}_{2}$-binding surface in the distal $\mathrm{C} 2$ domain is colored in blue. The locations of the lipid-binding pockets are distributed on different sides of the PI3KC2 $\alpha$ molecule in the closed conformation. 


\section{Reporting Summary}

Nature Research wishes to improve the reproducibility of the work that we publish. This form provides structure for consistency and transparency in reporting. For further information on Nature Research policies, see our Editorial Policies and the Editorial Policy Checklist.

\section{Statistics}

For all statistical analyses, confirm that the following items are present in the figure legend, table legend, main text, or Methods section.

$\mathrm{n} / \mathrm{a}$ Confirmed

$\bigotimes$ The exact sample size $(n)$ for each experimental group/condition, given as a discrete number and unit of measurement

\ A statement on whether measurements were taken from distinct samples or whether the same sample was measured repeatedly

The statistical test(s) used AND whether they are one- or two-sided

Only common tests should be described solely by name; describe more complex techniques in the Methods section.

Х A description of all covariates tested

\A description of any assumptions or corrections, such as tests of normality and adjustment for multiple comparisons

$\triangle$ A full description of the statistical parameters including central tendency (e.g. means) or other basic estimates (e.g. regression coefficient)

AND variation (e.g. standard deviation) or associated estimates of uncertainty (e.g. confidence intervals)

For null hypothesis testing, the test statistic (e.g. $F, t, r$ ) with confidence intervals, effect sizes, degrees of freedom and $P$ value noted Give $P$ values as exact values whenever suitable.

Х $\square$ For Bayesian analysis, information on the choice of priors and Markov chain Monte Carlo settings

$\bigotimes \square$ For hierarchical and complex designs, identification of the appropriate level for tests and full reporting of outcomes

$\triangle \square$ Estimates of effect sizes (e.g. Cohen's $d$, Pearson's $r$ ), indicating how they were calculated

Our web collection on statistics for biologists contains articles on many of the points above.

\section{Software and code}

Policy information about availability of computer code

Data collection Data for immunofluorescence images were acquired using FIJI Version : 2.1.0/1.53c

Quantiative values from gel were acquired using imageJ Version: 1.53

Data analysis All statistical tests were performed using Graphpad Prism9

X-ray diffraction data were processed using XDSAPP (Sparta et al., 2016)

Crystal structure determination/ refinement software package: PHENIX (1.18.2), Refmac (version 5.5 and higher) in CCP4 package (version

7.1), and coot (1.0.0).

EM particle autopicked used Gsutomatch-0.53

EM 2D class averages: RELION-2.0 and higher

Cross-linking MS/MS data were analyzed by using XlinkX standalone

Structures fitting to EM map were performed with Chimera (1.15.0) 
Policy information about availability of data

All manuscripts must include a data availability statement. This statement should provide the following information, where applicable:

- Accession codes, unique identifiers, or web links for publicly available datasets

- A list of figures that have associated raw data

- A description of any restrictions on data availability

PDB coordination in this study have been deposited in PDB data bank (pdb code: 7BI2, 7BI4, 7BI6, 7BI9)

Original EM micrographs were deposited in the EMPIAR (code: EMPIAR-10665)

CryoEM data were deposited in the PDB data bank (pdb code: EMD-12191)

Cross-linked peptides were provides in Supplementary Table 3.xlsx of the manuscript

All of statistical source data and unprocessed western blots were available in source data section of the manuscript

\section{Field-specific reporting}

Please select the one below that is the best fit for your research. If you are not sure, read the appropriate sections before making your selection.

$\bigotimes$ Life sciences $\quad \square$ Behavioural \& social sciences $\quad \square$ Ecological, evolutionary \& environmental sciences

For a reference copy of the document with all sections, see nature.com/documents/nr-reporting-summary-flat.pdf

\section{Life sciences study design}

All studies must disclose on these points even when the disclosure is negative.
Sample size Sample sizes were not chosen based on pre-specified effect size but selected based on commonly adopted standards in the field, resulting in statistically meaningful comparison. Sample size were corresponding to 20 images per sample for microscopy-based quantifications (Posor et al. 2013). Multiple independent experiments were carried out as detailed in the figure legends and Data reproducibility section within methods.
Data exclusions No samples were excluded from analysis.
Replication All experiments were carried out under standard and clearly defined conditions, and were replicated successfully by at least one researcher and all attempts of replication were successful. The number of replicates of each experiment is specified in the corresponding figure legend and data and reproducibility section within the Methods.

Randomization No animals have been used for this study, and No randomization was needed for the experiment with cultured cell line as cells were passaged in the same step from one parental cell dish for all groups in each experiment.

Blinding Immunofluorescence images were captured blindly by selecting cells in the DAPI channel or in the GFP channel (when performing knocked down and re-expressed experiments).

\section{Reporting for specific materials, systems and methods}

We require information from authors about some types of materials, experimental systems and methods used in many studies. Here, indicate whether each material, system or method listed is relevant to your study. If you are not sure if a list item applies to your research, read the appropriate section before selecting a response.

\begin{tabular}{l|l} 
Materials \& experimental system \\
\hline n/a & Involved in the study \\
\hline & $\bigotimes$ Antibodies \\
$\square$ & $\bigotimes$ Eukaryotic cell lines \\
$\searrow$ & $\square$ Palaeontology and archaeology \\
$\square$ & $\square$ Human research participants \\
$\searrow$ & $\square$ Clinical data \\
$\searrow$ & $\square$ Dual use research of concern
\end{tabular}

Methods n/a Involved in the study

$\triangle \square$ chip-seq

Х $\square$ Flow cytometry

Х $\square$ MRI-based neuroimaging

Antibodies

Antibodies used 
anti-GFP antibodies (Rabbit, Gulluni et al., 2017, Cancer Cell, 1:2000), TACC3 antibody (Rabbit, Cell Signaling \#8069, 1: 1000) Secondary antibody:

HRP-conjugated anti-Rabbit IgG light chain (1:5000, Jackson ImmunoResearch, 211-032-171)

Goat anti-mouse IgG (H+L) AF647 (1:400, Thermo Fisher, \#A21237)

Validation

$\mathrm{PI}(3,4) \mathrm{P} 2$ antibody and TACC3 antibody were validated by the manufacture's website anti-GFP antibody was validated in Gulluni et al., 2017

Eukaryotic cell lines

Policy information about cell lines

Cell line source(s)

Cos7 and HeLa cells were obtained from ATCC.

Authentication

Cell lines from ATCC are regularly authenticated by STR profiling and were used by us without further authentication.

Mycoplasma contamination

Cell lines were regularly tested for mycoplasm contamination and were not contaminated

Commonly misidentified lines (See $\underline{\text { ICLAC }}$ register)

No commonly misidentified cell lines were used in the study. 